\title{
Assessment of a 2D Synthetic Turbulence Method for Predicting the ACAT1 Fan's Broadband Noise
}

\author{
Carolin A. Kissner, Sébastien Guérin†, and Maximilian Behn ${ }^{\ddagger}$ \\ German Aerospace Center (DLR), Berlin, Germany
}

\begin{abstract}
Fan broadband noise, particularly rotor-stator-interaction noise, is an important noise mechanism in modern aircraft engines. However, its prediction is not trivial. Applied approaches range from fast but simplified analytical approaches to computationally demanding turbulence scale-resolving simulations. RANS-informed synthetic turbulence methods present a promising compromise between accuracy and cost. Two-dimensional simulations on a single streamline are especially attractive as they can be easily computed on conventional computers. While a good agreement with NASA SDT experimental data could be shown in the past, it can be questioned how representative a $2 \mathrm{D}$ simulation at an arbitrarily chosen streamline position can truly be since the flow and turbulence in the interstage region is highly dependent on the fan geometry and its operating conditions. In this paper, the authors propose a new, 3D-equivalent approach for setting up a $2 \mathrm{D}$ synthetic turbulence simulation. The results are compared to approaches that were applied for past investigations. In addition, a detailed comparison to the comprehensive, acoustic test data for the AneCom AeroTest 1 (ACAT1) transonic fan is shown. The good agreement between numerical and experimental data proves that a fast, two-dimensional synthetic turbulence method is indeed capable of accurate fan broadband noise predictions.
\end{abstract}

\section{Introduction}

Fan noise is understood to be an important noise source for an aircraft in flight, especially during the critical flight phase of approach.

While fan tones have been extensively studied both numerically and experimentally leading to the development of effective noise abatement measures, the progress on fan broadband noise reduction is less advanced. This can be partly attributed to the fact that turbulence, the key ingredient in the broadband noise generation mechanism, is challenging to predict and to model. Applied broadband noise prediction methods range from analytical to fully-scale resolving methods. On the one hand, analytical methods are fast and therefore suitable for optimizing fan designs but they are typically restricted to simplified flows and geometries. On the other hand, scale-resolving methods have the potential of capturing the physics most accurately but long computational times and large output data are significant drawbacks.

Synthetic turbulence methods offer a compromise between accuracy and cost. They can consider complex flows and geometries but are limited to the quality of (U)RANS simulations, which are used for imposing mean flow and turbulence characteristics. Most synthetic turbulence methods are hybrid approaches that tackle each aspect of the physical problem with a separate, highly specialized approach. For broadband rotor-stator-interaction noise, these methods generally work as follows: A CFD simulation is used to provide the mean flow and turbulence statistics. Turbulence is then synthesized upstream of the leading edges of the

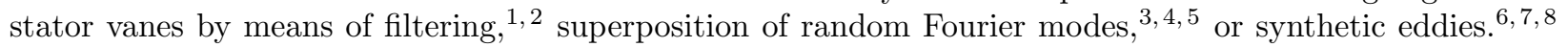
The synthesized turbulence is then convected in the mean flow by a Computational AeroAcoustics (CAA) code until it interacts with the leading edge of the blade geometry. The resulting noise can either be

\footnotetext{
${ }^{*}$ Research Engineer, Institute of Propulsion Technology, Department of Engine Acoustics, Müller-Breslau-Str. 8, 10623 Berlin, Germany, carolin.kissner@dlr.de.

${ }^{\dagger}$ Senior Research Scientist, Institute of Propulsion Technology, Department of Engine Acoustics, Müller-Breslau-Str. 8, 10623 Berlin, Germany.

${ }^{\ddagger}$ Research Engineer, Institute of Propulsion Technology, Department of Engine Acoustics, Müller-Breslau-Str. 8, 10623 Berlin, Germany.
} 
analyzed by propagating the sound waves from the source to the sensors in the far field or by using the pressure fluctuations on the vane surface as an input for a Ffwocs-Williams-Hawkings technique.

Currently, both two-dimensional and three-dimensional synthetic turbulence methods are commonly used. Three-dimensional approaches are advantageous as they can consider complex, 3D flows and enable the realization of fully anisotropic turbulence as shown by Gea-Aguilera et al $\underline{9}^{[}$They can also consider vane geometries that change significantly in the spanwise direction, e. g. due to leading edge serrations as demonstrated in several works $10[11[12] 13$ However, 3D methods tend to be expensive. Possible strategies aimed at mitigating simulation times include:

- The simulation domain can be limited to one or a few stator channels. The implications are discussed by Reboul et al! 13

- The simulation domain can be limited to a spanwise section as was the case for Clair et al.'s $\mathrm{s}^{10}$ study of leading edge serrations.

- The grid design can be coarser. The impact of a reduction in radial points has been studied by Reboul et al 13

- The sampling time can be shortened, oftentimes limited to just two rounds. This is probably the most common strategy applied by nearly all authors.

- For methods relying on the summation of Fourier modes, turbulent forcing can be reduced to two or even just one velocity component. Gill et al ${ }^{14}$ concluded that considering only the transverse velocity component is adequate for single, symmetric airfoils. In a more recent work, Cader et al $\frac{15}{\sqrt{15}}$ have applied two- and three-component formulations for turbulence generation for a real fan stage, i. e. the ACAT1 fan at approach conditions.

All of these measures have a potential impact on the acoustic results. Compared to 3D approaches, 2D methods are faster and can typically be computed on a few cores of a conventional computer. It is thus not problematic to be more conservative in mesh design or to increase the sampling duration. However, the question remains if a $2 \mathrm{D}$ simulation on one streamline can truly be representative for the entire duct. In the past, Wohlbrandt et al $\frac{16}{16}$ investigated the impact of cyclostationarity on fan broadband noise for the NASA Source Diagnostic Test (SDT) Fan and performed their 2D studies at a streamline located at the midspan position of the stator. The numerical results agreed well with experimental data. More recently, Kissner et al $17 \mid 18$ studied a more modern fan using three 2D simulations at $20 \%, 50 \%$, and $80 \%$ of the stator height to get a more comprehensive fan noise prediction. For the investigated configuration at approach operating conditions, the averaged results deviated only slightly from the simulation at the stator midspan position. The plausibility of the results was checked by comparing the numerical data to scaled, experimental data of the NASA SDT fan. However, choosing one supposedly representative streamline position independent of fan geometries and operating points is arbitrary and the quality of results thus a matter of experience and luck.

In this paper, the authors propose a new, so-called 3D-equivalent method with the aim of ensuring that the fan broadband noise prediction of a $2 \mathrm{D}$ synthetic turbulence method is representative for the entire $3 \mathrm{D}$ duct. The new approach relies on a detailed analysis of the flow and turbulence in a CFD simulation near the stator leading edge to determine a streamline position and a representative turbulence. The quality of the noise prediction is assessed by comparing the numerical results to past approaches. Thus, simulations will be performed at $20 \%, 50 \%$, and $80 \%$ of the stator height using the local flow and turbulence variables. In addition, the numerical results are compared in detail to experimental data of the transonic ACAT1 fan at approach conditions. The assessment is made in terms of sound pressure and sound power based on analyses of overall levels, frequency spectra, and circumferential mode content.

The paper is structured as follows: In the first section, a general overview of the used method is given, the used CFD input is discussed, and an example of a setup for a 2D simulation for fan broadband prediction is shown. The next section concerns the 3D-equivalent technique and briefly revisits past approaches. The inputs and results of the different approaches are compared. In the final section, the experimental and numerical data are examined in terms of sound power and sound pressure. 


\section{Overview and setup of the $2 \mathrm{D}$ fan broadband prediction}

This section gives a short, general overview of the method. Then the used CFD input is discussed and a typical setup of a 2D fRPM-fan simulation is shown.

The fRPM-fan simulations featured in this paper were performed for the ACAT1 fan configuration at approach operating conditions. The scaled testing fan has a diameter of $85 \mathrm{~cm}$ and is transonic. The design pressure ratio is about 1.42 and the design bypass ratio is about 8 . The fan has 20 rotor blades, 44 outlet guide vanes (OGV's), and 44 engine support stator vanes. The approach conditions were chosen to match approach testing conditions for the Sea Level Static working line of the so-called short gap configuration at the Universal Fan Facility for Acoustics. Details regarding all tested certification operating points are listed by Guérin et al $\frac{19}{19}$

\section{A. Method overview}

The fRPM-fan method refers to the fRPM-based synthetic turbulence method that is specifically suited for fan applications. Note that the overview given here is therefore not representative for all fRPM-based techniques. The method is essentially a hybrid method consisting of three separate methods: the Computational Fluid Dynamics (CFD) method, the fast Random Particle Mesh (fRPM) method, and the Computational AeroAcoustics (CAA) method.

A RANS or URANS simulation computes the mean flow and turbulence statistics required as inputs for the fRPM and CAA methods. The fRPM method is a fast implementation of the Random Particle Mesh (RPM) method, ${ }^{2}$ which uses recursive filters on a Cartesian grid to speed up simulations $\stackrel{20}{2}$ The fRPM method for fan applications does not directly model source terms but synthesizes time-space-dependent turbulence upstream of the stator row. It works as follows: White noise is scaled by a local variance, i.e. a turbulent kinetic energy, and then spatially filtered by a local length scale. Several Gaussian filters of different filter lengths can be superposed in order to realize a target spectrum, typically a von Kármán spectrum.21 The variance of each filter depends on an analytical weighting function.

The convection of the synthesized turbulence, the sound generation, and the sound propagation was computed using the CAA solver PIANO ${ }^{22}$ In this work, the linearized Euler equations (LEE) are solved. A fourth order low-dispersion low-dissipation Runge-Kutta scheme ${ }^{23}$ was used for the time integration and the dispersion-relation-preserving finite difference scheme by Tam and Webb, $\stackrel{24}{,}$ for the spatial discretization. The fluctuating velocity fields are solenoidally coupled into the CAA domain by adding a relaxation term to the momentum equations as proposed by Ewert et al.25

The sound is generated when the turbulence interacts with the leading edge of a stator vane. The sound waves then propagate to sensor positions in the far field region to determine sound power levels. Note that the method is technically not restricted to the consideration of rotor-stator-interaction noise, which results from the interaction of the wake turbulence with the stator leading edges. In fact, broadband noise resulting from the interaction of ingested turbulence or boundary layer turbulence with the leading edges of the stator can also be considered.

\section{B. CFD input}

For this work, the turbulence statistics and mean flow were extracted from a URANS simulation. Note that the used fRPM-fan approach does not require an unsteady solution. A steady solution would be suitable as well. The authors chose to use the URANS simulation for two reasons: 1.) The fRPM-fan method in combination with the URANS simulation allows for the prediction of the entire rotor-stator-interaction noise: tones (URANS) and broadband noise (fRPM-fan). 2.) The same simulation can be used for future investigations focusing on the expansion of the fRPM-fan method to three-dimensional space or studying cyclostationary and anisotropic turbulence in the interstage region. Conversely, this also means that if tones are computed numerically, it automatically produces all inputs necessary for the simulation of the broadband noise mechanism.

The URANS simulation of the ACAT1 was performed using the DLR in-house CFD solver TRACE. 26 The SSG/LRR- $\omega$ full Reynolds stress turbulence model (RSTM) ${ }^{27}$ was applied. One advantage of this model is that it computes more realistic, anisotropic turbulence, which will be interesting for future investigations. The other advantage is that it is particularly suited for considering ingestion turbulence. Many two-equation turbulence models have an unrealistically high dissipation rate causing the prescribed inlet turbulence to 
dissipate completely before reaching the interstage section of a fan stage. This is particularly critical if the ingested turbulence does not have a negligibly small turbulence intensity or turbulent length scale. In this case, turbulence spectra measured by hot wire probes were fitted with von Kármán spectra to determine turbulence statistics. Outside of the boundary layer, the turbulence intensity was estimated to about $0.3 \%$ and the turbulent length scale, to about $0.04 \mathrm{~m}$. This turbulence along with flow conditions extracted from pressure rakes in the inlet were prescribed to match test conditions and ensure the correct thickness of the boundary layer.

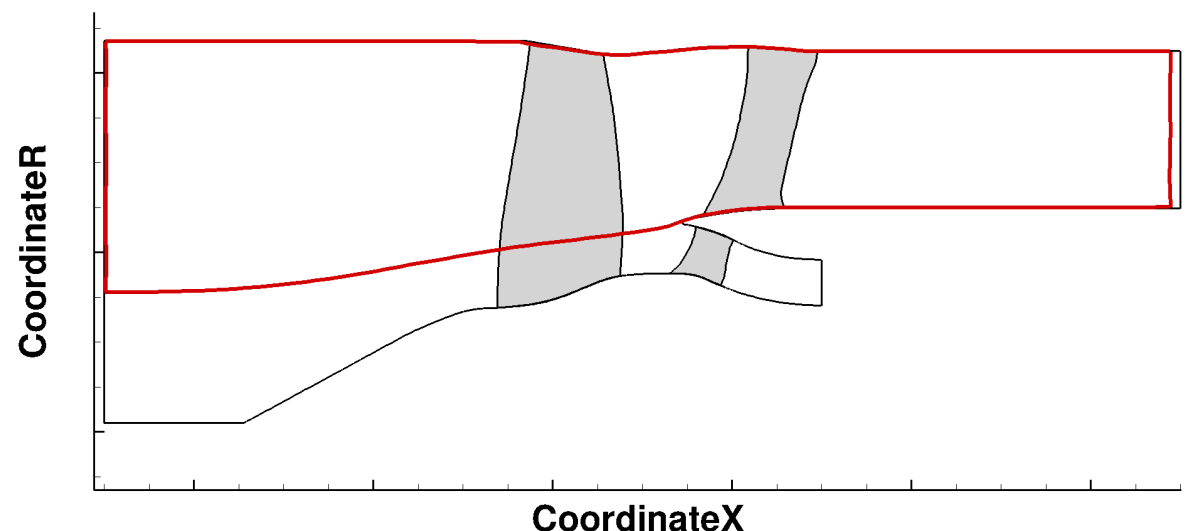

Figure 1. URANS Domain outlined in red

The computation domain was reduced to the bypass as the investigation will focus on the interaction noise between the rotor blades and the OGV's. The bypass and core flows were separated by computing a streamline at the stator hub for a RANS simulation performed at the same operating conditions. The reduced domain is shown in red in Fig. 1. Since phase-lag conditions in combination with the chosen turbulence model are not yet available, periodic boundary conditions are applied. The computation domain thus contains 5 rotor blades and 11 OGV's. During the mesh generation process, great care was taken to fully resolve wakes and boundary layers. The resulting mesh contains about 30 million cells, which should suffice for the prediction of fan tones.

To ensure that the reduction of the domain to the bypass flow does not significantly alter the flow and turbulence in the interstage region, which is critical for subsequent broadband noise predictions, the turbulence of three RANS simulations were analyzed on an extraction plane close to the rotor trailing edge. The three investigated RANS simulations include a full fan stage simulation using the Menter SST k- $\omega$ turbulence model $[28$ a bypass-only simulation using the Menter SST k- $\omega$ turbulence model, and a bypassonly simulation using the SSG/LRR- $\omega$ full Reynolds stress turbulence model. The latter simulation was used to set up the subsequent URANS simulation. While the changes in mean flow are negligible, some differences can be observed in the turbulence characteristics shown in Fig. 2. All of the turbulent length scales (TLS) shown in this paper were determined using a spectral averaging technique $\frac{16}{17}[18$ The turbulent kinetic energy (TKE) is slightly higher near the tip region for the full fan stage simulation, while the choice of turbulence model has little impact on the TKE. The SSG/LRR- $\omega$ full Reynolds stress turbulence model does, however, produce a slightly larger turbulent length scale (TLS), particularly in the midspan region. This slightly higher TLS mainly explains the slightly higher turbulence levels of less than $2 \mathrm{~dB}$ in the radially averaged transverse velocity frequency spectrum. The frequency peak was also slightly shifted towards a lower frequency. For the simulations using the Menter SST k- $\omega$ turbulence model, the radially averaged turbulence spectra are nearly identical despite some differences in the radial distribution of TKE and TLS. This indicates that the overall turbulence in the interstage region remains almost unchanged due to reduction of the simulation domain to the bypass flow.

Assessing the convergence of the URANS simulation proved to be challenging as the simulation for this configuration at the investigated approach conditions features a fluctuating leading edge (LE) separation on the rotor blades. This LE instability varies in its severity but effectively spans the entire radial height of the computational domain. This causes the rotor wakes to exhibit a fluctuating behavior as can be seen in Fig. 3. While this phenomenon of LE separations is not untypical for off-design operating points, it is questionable if the structure can be reproduced correctly by this simulation setup. The instability is forced 


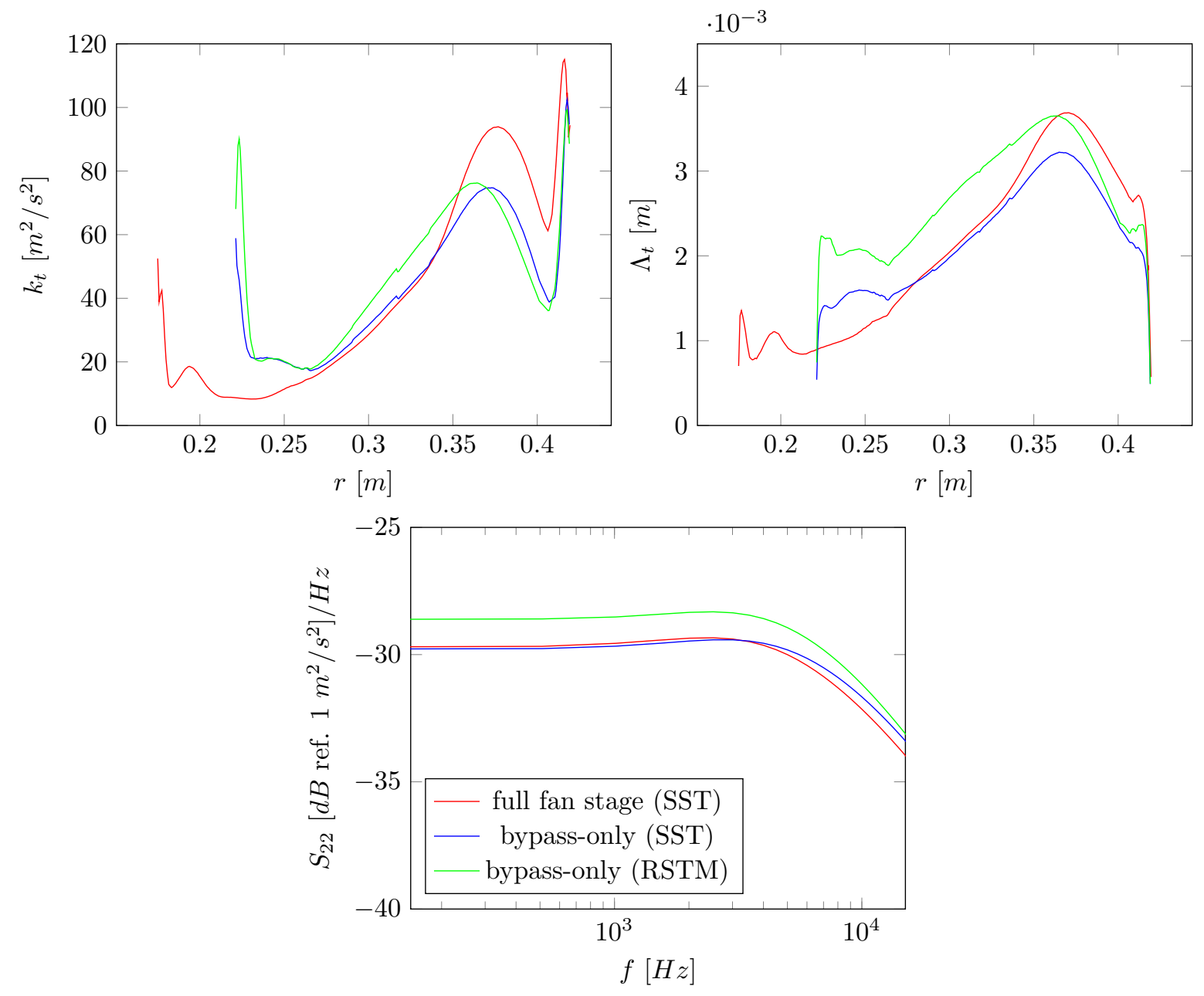

Figure 2. Comparison of the interstage turbulence for full fan stage and bypass-only RANS simulations featuring the Menter SST k- $\omega$ and the SSG/LRR- $\omega$ full Reynolds stress turbulence models: Radial distributions of turbulent kinetic energy $k_{t}$ and turbulent length scale $\Lambda_{t}$ as well as radially averaged upwash velocity frequency spectra are shown. 


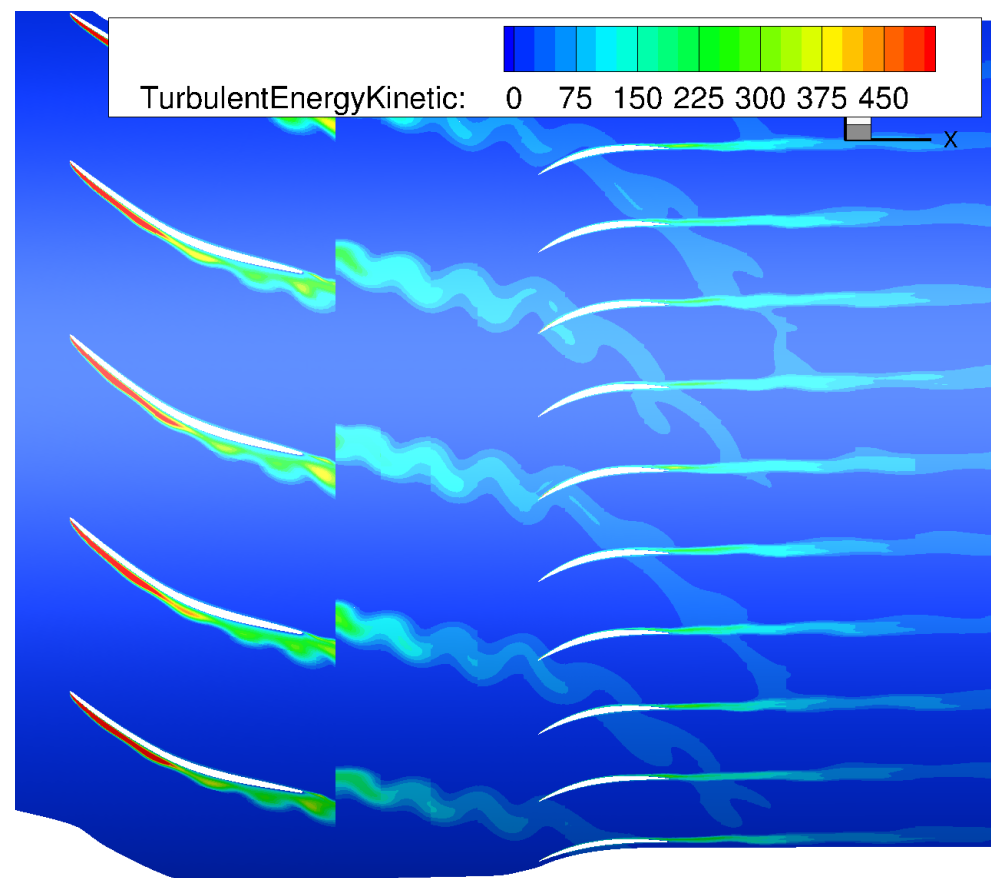

Figure 3. Instantaneous snapshot showing the fluctuating leading edge seperation on the rotor blades at one radial position

into a periodic pattern due to the imposed boundary conditions and Fourier harmonics are only output at rotor harmonics in the stator domain, even though the unsteadiness is probably neither periodic or related to rotor harmonics into such a pattern. Nonetheless, the authors chose to look at two criteria to determine whether the simulation is suited for broadband noise prediction: tones in the bypass duct and interstage turbulence characteristics.

The fan features a cut-off design at the blade passing frequency (BPF). For the second and third BPF's, exactly one interaction mode of azimuthal mode order $m$ is expected to be cut-on respectively according to the Tyler and Sofrin (TS) rule $\frac{29}{29} m=4$ at BPF 2 and $m=-16$ at BPF 3. When extracting the azimuthal modes using the extended triple plane pressure mode matching technique of Wohlbrandt et al., 30 not only the TS-acoustic modes are detected. In addition, the azimuthal mode order 0 is detected with a similar magnitude as the acoustic modes. Modes that are multiples of four are also present but their magnitude is lower than for the acoustic modes. This can most likely be attributed to the LE separation, which is forced into periodic, rotor-harmonic pattern by the simulation setup. While it can be seen in Fig. 4 that the azimuthal modes of order 0 fluctuate significantly, the interaction modes show a convergent behavior. After 15 periods ( 1 period $=0.25$ revolution), the changes in sound power are small and levels converge to about $117 \mathrm{~dB}$ at BPF 2 and $106 \mathrm{~dB}$ at BPF 3.

In a second step, the turbulence was analyzed on a slice near the stator leading edge for four solutions that were output at computational times greater than 15 periods to examine the impact of the LE separations on the interstage turbulence and flow. The analysis showed essentially no differences in the mean flow and only some differences in the turbulence characteristics as shown in Fig. 5 The variations in TLS and TKE are greatest near the tip, where the influence of the LE instability is largest. Despite this, little difference in the overall turbulence can be observed. The levels of the radially averaged transverse velocity frequency spectra deviate by less than $1 \mathrm{~dB}$ from the mean spectrum. Since the transverse velocity component is widely regarded as the most critical for the broadband rotor-stator-interaction noise mechanism, the predicted broadband noise levels are expected to vary by less than $1 \mathrm{~dB}$. Due to small, expected overall impact of the fluctuating wakes and the convergence of the interaction modes, the authors chose to use solution $\mathrm{A}$ as the input for the following fRPM-fan simulations. 


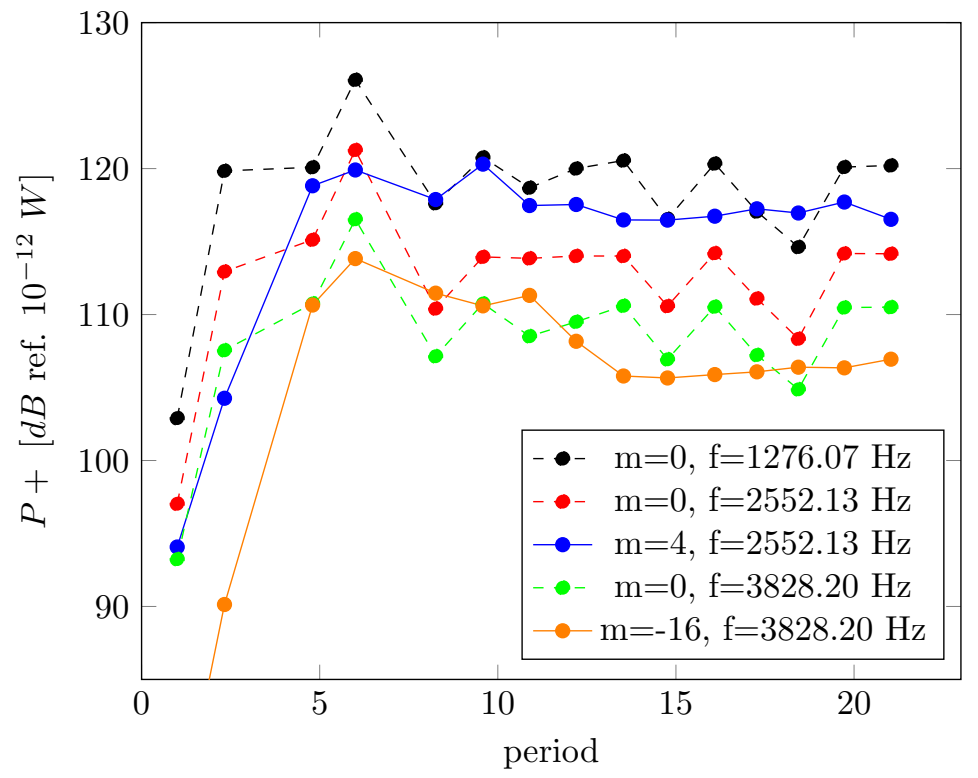

Figure 4. Cut-on azimuthal modes in the bypass duct: Tyler and Sofrin acoustic modes are marked by solid lines.

\section{FRPM-fan simulation setup}

An exemplary fRPM-fan simulation setup is shown in Fig. 6. The shown setup contains 11 stator vanes, thus only a quarter duct was simulated. Sensor positions and the vortex source and sink regions are shown in the figure.

The vortex source is an fRPM region, where the synthesized turbulence is injected into the CAA domain. The fRPM patch is rotated into the direction of the flow and is designed so that the injected turbulence interacts with only one stator vane. The other stator vanes are present to ensure the correct radiation of the produced sound. In the subsequent data processing, it will be assumed that the vanes are acoustically uncorrelated, which is an assumption that was shown to be appropriate by Wohlbrandt et al ${ }^{16}$ Since all injected turbulent structures span less than one pitch, this assumption is thought to hold true for the presently investigated case as well.

Downstream of the stator vanes, the synthesized vortices tend to interact with the stator wakes resulting in hydrodynamic pressure fluctuations. As the LEE's have no damping term and only the acoustic pressure fluctuations are of interest, the vortex sink, located downstream of the stator vanes, uses the LEE-relaxation method to remove the vortices in that region.

Sensors are located between the leading edges of vortex source and stator leading edge to monitor the correct realization of the imposed turbulence. Up- and downstream of the stator vanes, equally spaced sensors are placed to allow for the computation of the sound power levels under the assumption that the results at a given streamline position are representative for the entire duct 16$] 17][18$

Near the in- and outlet boundaries (up- and downstream of the respective sensors), sponge zones and grid stretching were used to avoid reflections at the boundaries. In circumferential direction, a periodic boundary condition was imposed.

For the mesh design, the vortices have to be resolved in regions upstream of and at the stator vanes, where the injected turbulence has to be propagated. In other areas of the mesh outside of the sponge zones, the acoustic resolution is essential for the mesh design to ensure the correct propagation of sound waves from the source at the leading edges to the sensor positions. For the $2 \mathrm{D}$ simulations, a maximum target frequency of $10 \mathrm{kHz}$ was chosen. The typical 2D mesh size was between 9208 and 12280 cells per passage. For performing 1,000,000 time steps, which is equivalent to 4.84 full rounds, about 200 CPU hours per 100,000 cells were required using Intel(R) Xeon(R) CPU E7-4830 v3 @ 2.10GHz. The quarter duct simulations could therefore be computed on a few CPU's of a conventional computer in less than 24 hours. 

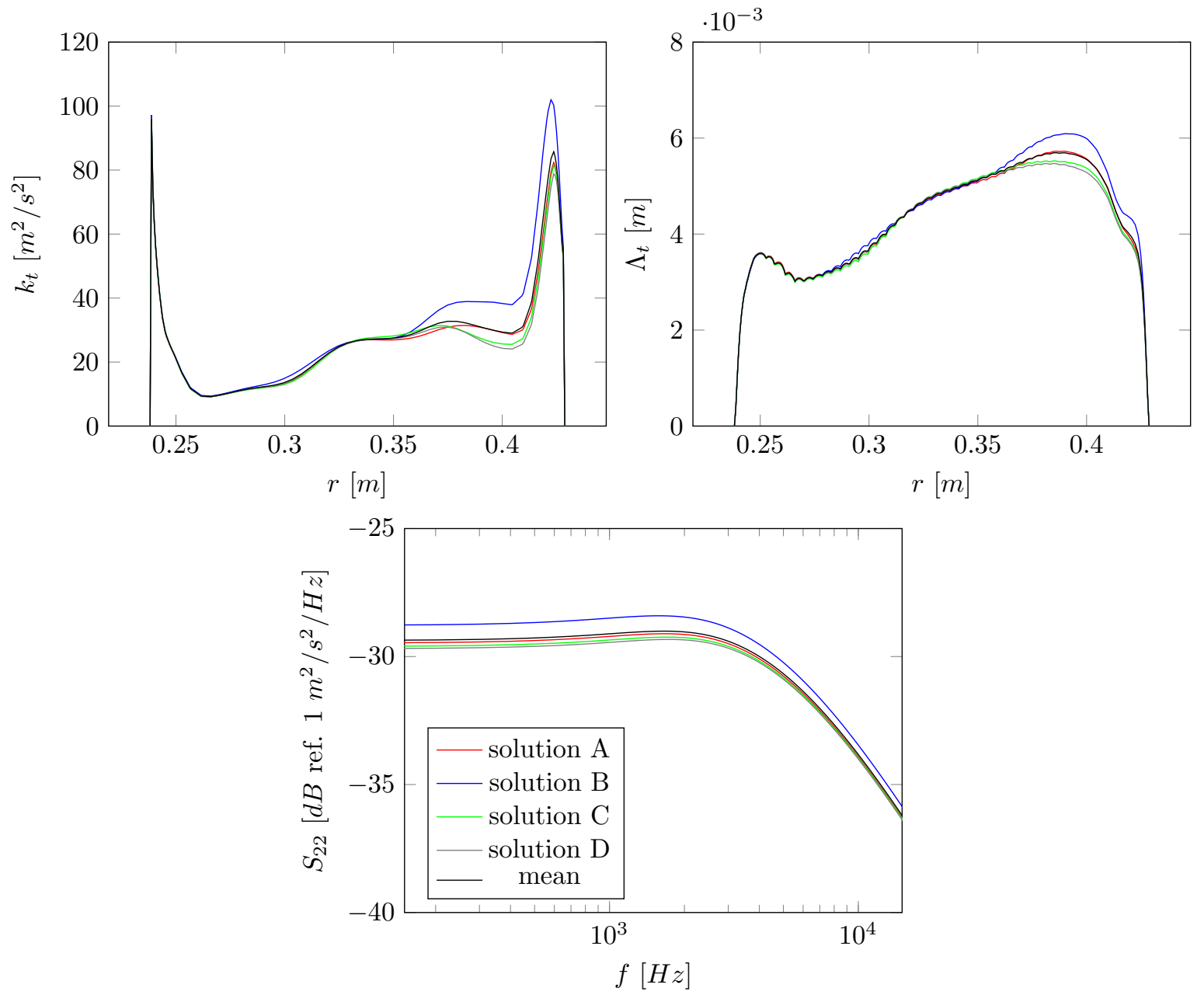

Figure 5. Comparison of turbulence characteristics for different time solutions of the same URANS simulation: Radial distributions of turbulent kinetic energy $k_{t}$ and turbulent length scale $\Lambda_{t}$ as well as radially averaged upwash velocity frequency spectra are shown. Mean values are denoted by black lines. 


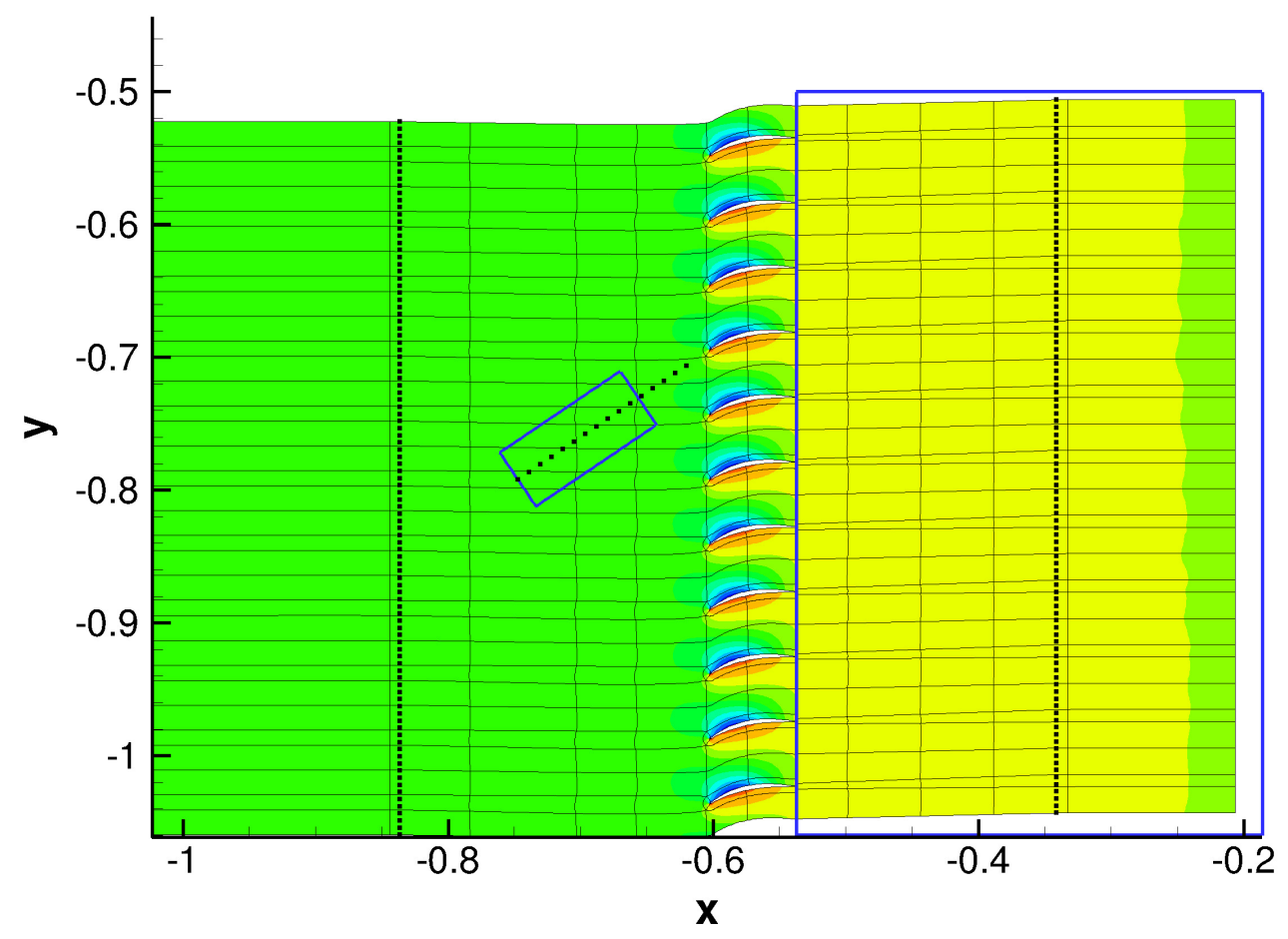

Figure 6. CAA Setup containing 11 stator vanes: Sensor positions are indicated by black marks, while the vortex source and sink regions have blue edges.

\section{Acoustic mesh resolution}

To ensure the propagation of sound without dissipation effects, azimuthal modes are resolved up to the maximum target frequency of $10 \mathrm{kHz}$. A maximum cell size is computed based on critical axial wavelengths for the up- and downstream propagation of sound. Circumferential wavelengths are less critical, particularly at frequencies below $10 \mathrm{kHz}$ and since the circumferential cell size is defined by the more restrictive criterion of turbulence resolution.

For the determination of the required acoustic resolution, a 2D, low swirl formulation, where azimuthal modes are the only relevant eigenfunctions, was used. Formulas and involved assumptions are listed and discussed in greater detail by Guérin et al ${ }^{[19}$ The wavelength was determined as follows:

$$
\Lambda_{x, m}^{ \pm}=\frac{1-M_{x}^{2}}{k \delta_{m}} \frac{2 \pi}{-M_{x} \pm \alpha_{m}}
$$

where the wavenumber $k$ depends on the maximum frequency $k=\frac{2 \pi f_{\max }}{c}$ and $\delta_{m}$ relates to the swirl Mach number $M_{s}$ as follows:

The cut-on factor is defined as:

$$
\delta_{m}=1-\frac{m M_{s}}{k R}
$$

$$
\alpha_{m}=\sqrt{1-\frac{\left(1-M_{x}^{2}\right)}{\delta_{m}^{2}} \frac{m^{2}}{(k R)^{2}}} .
$$

The aim was to resolve even the smallest wavelength of all cut-on modes in the target frequency range with a least 7 points per wavelength (PPW). This complies with the theoretical resolution limit of 5.4 PPW for a Dispersion-Relation-Preserving (DRP) scheme ${ }^{31}$ Thus, the maximum allowed cell size was computed as follows:

$$
\delta x \leq \min \left(\frac{\Lambda_{x, m}^{ \pm}}{P P W}\right) .
$$

For all simulations, the critical cell size, which represents a maximum cell size limit, to resolve the acoustics upstream of the stator vanes was greater or equal to $0.003 \mathrm{~m}$ and to resolve the acoustics downstream 
of the stator vanes, greater or equal to $0.006 \mathrm{~m}$. These theoretical limits were never reached, particularly downstream of the stator vanes. The cell size in the acoustically relevant regions was influenced by smaller cell size requirements to resolve turbulent structures in the region, where turbulence is injected and propagated. As the high-order schemes employed by the CAA code require small stretching ratios, the cell size could only be moderately increased in the acoustically relevant regions. The maximum cell size was therefore about $0.003 \mathrm{~m}$. In the upstream direction, the largest cell size was typically smaller as most of that area was designed to resolve turbulence. For a maximum cell size of $0.003 \mathrm{~m}$, the resolved acoustic azimuthal modes are shown in Fig. 7. The modes outside of the cones are cut-off and white regions within the cut-on cone are under-resolved. It is thus proven that the acoustics are well resolved up to at least the target frequency of $10 \mathrm{kHz}$ up- and downstream of the stator vanes.
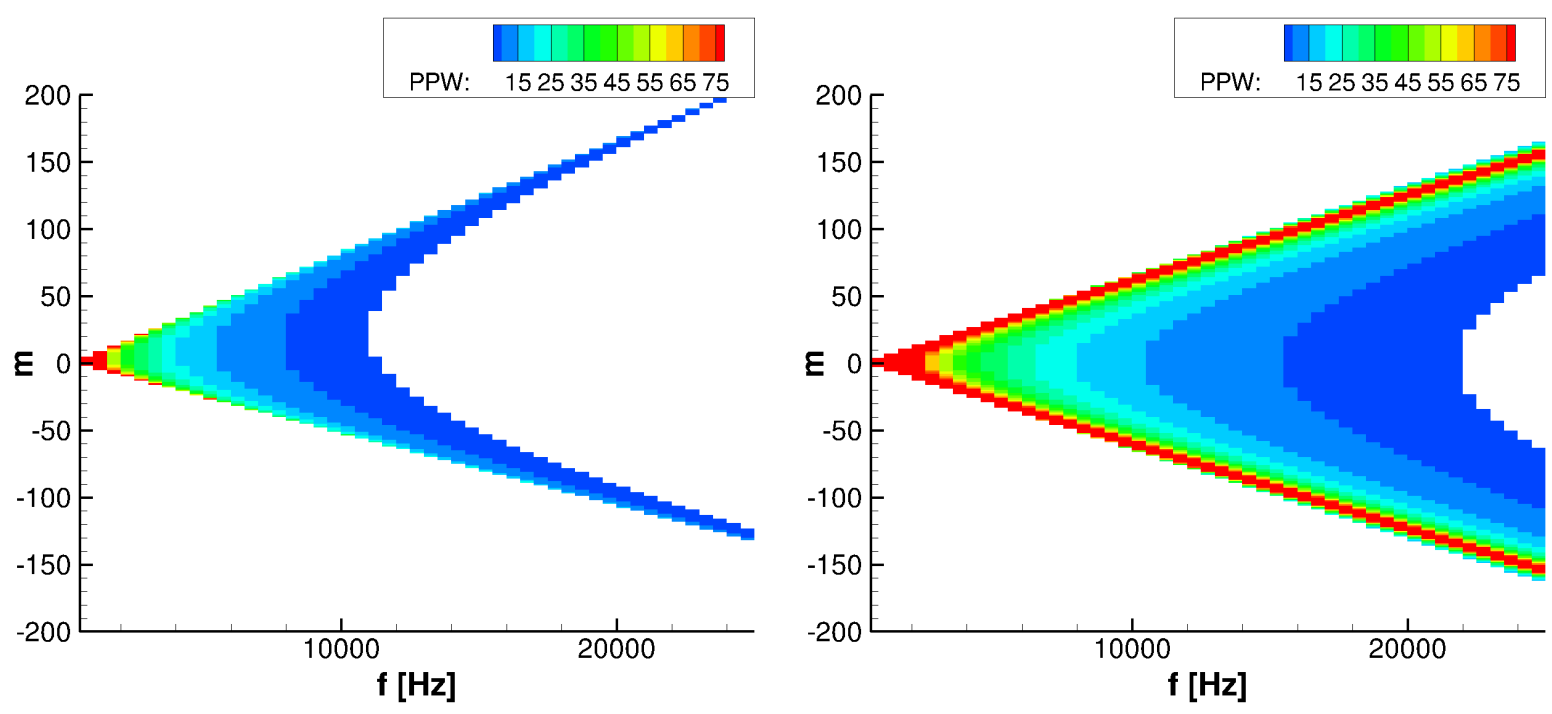

Figure 7. Resolved acoustic modes for the used, maximum cell size of 0.003m up- (left) and downstream (right) of the stator vanes: White regions within the cut-on domain mark modes that are resolved with less than the target 7 PPW.

\section{Turbulent mesh resolution}

To synthesize a realistic turbulence spectrum such as the von Kármán spectrum, Gaussian filters are superposed to approximate a target spectrum. Essentially, a length scale is prescribed for each Gaussian filter and an analytical weighting function is applied to each respective filter to determine the variance of the filter. Further details regarding this technique were described in the work of Wohlbrandt et al.21

In order to find the most computationally efficient settings that fulfill given accuracy targets, an optimization routine was employed. Given inputs include turbulence and flow characteristics (TKE, TLS, and mean flow velocity) to be realized, the maximum target frequency $(10 \mathrm{kHz})$, and the maximum deviation from the target spectrum at each frequency (between $-0.1 \mathrm{~dB}$ and $1.0 \mathrm{~dB}$ ). The following constraints have to be considered:

- The maximum allowed cell size must be smaller than the cell size required for resolving the acoustic waves.

- The minimum, permissible number of Gaussian filters is four.

- The smallest Gaussian length scale must not be smaller than twice the cell size.

- The largest Gaussian length scale must not exceed the patch dimensions in any direction.

The optimization routine then determines the optimal settings in terms of the minimum grid size, minimum Gaussian length scale, maximum Gaussian length scale, and the number of Gaussian filters. Four Gaussian filters were used for all simulations and the maximum allowed cell size in order to ensure a correct turbulence realization ranged between $0.0023 \mathrm{~m}$ and $0.0026 \mathrm{~m}$. 


\section{Fan noise prediction for three different 2D approaches}

In this section, the new, 3D-equivalent method is discussed. The approach based on multiple streamlines, hereafter referred to as multi-span approach, as well as the the approach based on a simulation at $50 \%$ of the stator height, hereafter referred to as midspan approach, are recapped. The imposed turbulence and flow is then compared for the different simulations. Finally, the predicted sound powers are compared and the findings are summarized. Note that in this section, all discussed simulations were performed for a quarter duct, i. e. containing only 11 stator vanes.

\section{A. 3D-equivalent approach}

As previously mentioned, one of the challenges of setting up a 2D synthetic turbulence method is to decide on a streamline position and turbulence characteristics in the hopes that the $2 \mathrm{D}$ simulation is representative for the entire 3D fan stage. In the past, this decision has been rather arbitrary. In this paper, a new method is proposed. It relies on a detailed analysis of the turbulence and flow in the fan interstage region to determine the most representative streamline and turbulence before setting up the $2 \mathrm{D}$ simulation. The aim is to achieve an accurate broadband noise prediction using the 2D fRPM-fan method for every fan geometry and every operating point in future works.

In a first step, a slice is extracted from a three-dimensional (U)RANS simulation in the interstage region, preferably close to the stator leading edge. All presented analyses were performed for the slice marked in red in Fig. 8. Analyses were performed on the other slices in order to ensure the consistency of the method.

Secondly, flow and turbulence characteristics are circumferentially averaged.

In the next step, an equivalent flow is determined and matched with a streamline location. The radial flow averaging relies on the fact that pressure fluctuations of the broadband rotor-stator-interaction noise mechanism are proportional to the squares of mean flow velocity, mean density, and fluctuating velocity:

$$
p^{\prime 2} \propto \rho_{0}^{2} u_{0}^{2} u^{\prime 2}
$$

For mean flow components involved in this noise source, the following averaging technique was employed:

$$
\overline{\rho_{0}^{2} u_{0}^{2}}=\frac{1}{n} \sum_{i=1}^{n} \rho_{0}\left(r_{i}\right)^{2} u_{0}\left(r_{i}\right)^{2},
$$

where $\mathrm{n}$ is the number of radial positions. For the resulting radially averaged flow $\overline{\rho_{0}^{2} u_{0}^{2}}$, a matching streamline position is determined. For the investigated case, the streamline position was located at $57.14 \%$ of the stator height. Therefore, the mean velocity $u_{0}$ is equal to $128.89 \mathrm{~m} / \mathrm{s}$ and the mean density $\rho_{0}$ is equal to $1.19 \mathrm{~kg} / \mathrm{m}^{3}$. The streamline position is shown in Fig. 8. The black squares indicate the position corresponding to the mean radial flow at each slice position. It shows that the positions do not deviate much from the chosen streamline, which indicates that the chosen approach is consistent.

The turbulence is radially averaged using the same scaling law of Eq. 5 as for the mean flow averaging. Here, it is assumed that the transverse velocity component is the most critical. This assumption is made in analytical, Amiet-based ${ }^{32}$ models and has also been numerically confirmed for symmetric airfoils $\frac{14}{4}$ The radially averaged upwash velocity frequency spectrum is determined as follows:

$$
\overline{S_{22}(f)}=\frac{\frac{1}{n} \sum_{i=1}^{n} \rho_{0}\left(r_{i}\right)^{2} u_{0}\left(r_{i}\right)^{2} S_{22}\left(r_{i}, f\right)}{\overline{\rho_{0}^{2} u_{0}^{2}}} .
$$

The total averaged spectrum is shown in black in Fig. 9. For a more in-depth analysis, averaged turbulence spectra can be determined for each turbulence component - wake, background, or boundary turbulence by only considering its respective contribution at each radial position. The resulting spectra hint at the expected contribution of each turbulent component to the overall sound generation mechanism. For the investigated case, the total turbulence is composed of:

- $75.93 \%$ wake turbulence,

- $23.26 \%$ boundary layer and boundary layer/tip vortex interaction turbulence,

- and $0.81 \%$ background turbulence. 


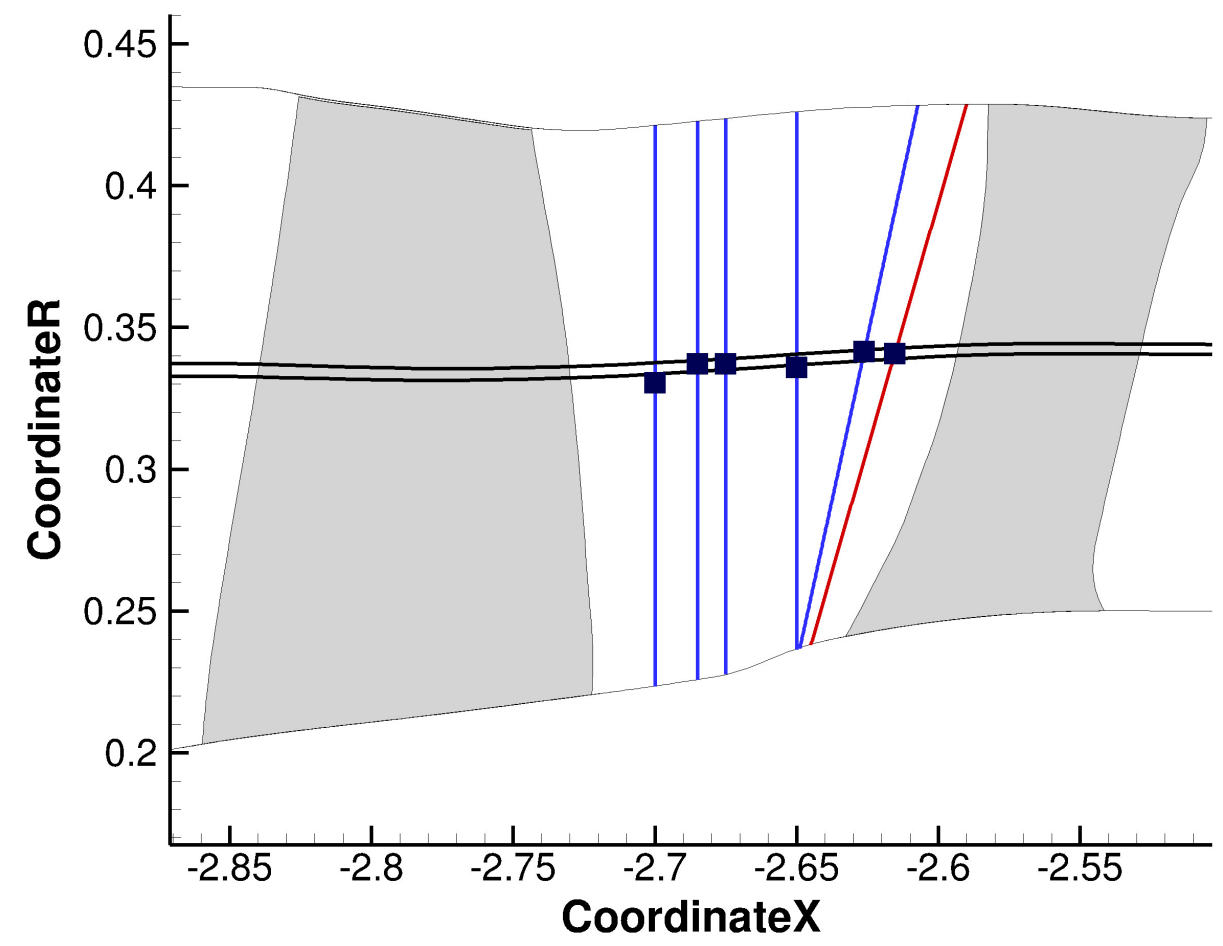

Figure 8. Streamline and extraction planes: The location of the analyis plane near the stator LE is marked in red. The black sqares indicate the respective positions associated with the radially averaged mean flow.

The spectral shapes and frequency peaks relating to the main turbulence contributors (wake and boundary layer) are very similar. The frequency peak of the background turbulence spectrum is shifted to a lower frequency due to the larger TLS of about $0.04 \mathrm{~m}$ in the background turbulence. However, since the contribution of the background turbulence is small, the shape of the total spectrum is shaped like a von Kármán spectrum. It is therefore not necessary to consider cyclostationary effects as opposed to the work presented by Kissner et al $17 \mid 18$

To determine the TKE and TLS of the radially averaged turbulence spectrum $\overline{S_{22}}$, it is fitted to a von Kármán using the previously determined mean flow velocity $u_{0}$ of $128.89 \frac{\mathrm{m}}{\mathrm{s}}$. The resulting TKE is equal to $22.78 \frac{\mathrm{m}^{2}}{\mathrm{~s}^{2}}$ and the resulting TLS is equal to $0.0046 \mathrm{~m}$.

In the last step, the 2D fRPM-fan simulation is setup by using the determined streamline at the position of the radially averaged flow and by imposing the TKE and TLS on the fRPM vortex source. Thus, the simulation is performed using a $3 \mathrm{D}$-equivalent flow and a $3 \mathrm{D}$-equivalent turbulence.

\section{B. Multi-span approach}

As shown by Kissner et al. $\frac{18}{18}$ another technique is to consider multiple streamlines. The sound power is then determined by averaging the turbulent intensities of all three simulations thus resulting in an averaged sound power spectrum. In the mentioned work, streamlines at the stator heights of $20 \%, 50 \%$, and $80 \%$ were chosen. The results of the averaged solution deviated only slightly from the results at $50 \%$ of the stator height. In addition, the plausibility of the results was proven by a comparison to scaled, experimental NASA SDT data. In this paper, the same positions were investigated but no cyclostionarity was considered as the background turbulence was estimated to be negligible (see previous Subsection).

\section{Midspan approach}

The midspan approach predicts fan broadband noise on a streamline positioned at $50 \%$ of the stator height. This approach was successfully used by Wohlbrandt et al $\frac{[16}{[1}$ and good agreement with experimental data was shown. 


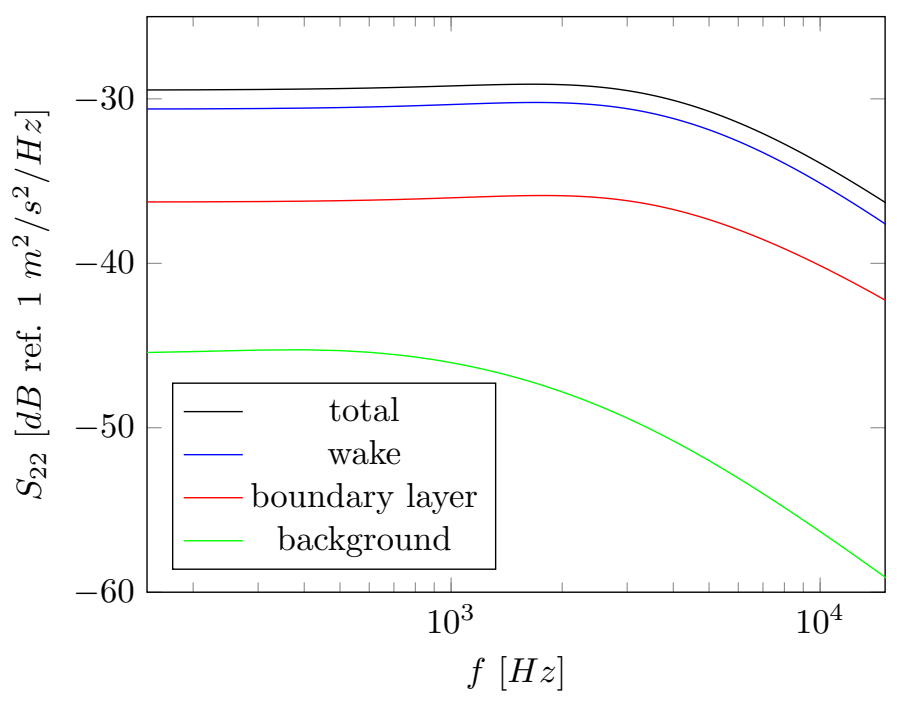

Figure 9. Radially averaged upwash velocity frequency spectra showing the total spectrum and the contribution of different turbulence components

Conversely to that work, no cyclostationarity was considered here for previously stated reasons.

\section{Comparison of imposed flow and turbulence}

Four 2D fRPM-fan simulations were performed to compare the different techniques for setting up 2D simulations:

- at $20 \%$ stator height (multi-span approach),

- at $50 \%$ stator height (multi-span and midspan approach),

- at $57 \%$ stator height (3D-equivalent approach),

- and at $80 \%$ stator height (multi-span approach).

Table 1. Comparison of turbulent kinetic energy $k_{t}$, turbulent length scale $\Lambda_{t}$, and mean flow velocity $u_{0}$

\begin{tabular}{cccc} 
& $u_{0}\left[\frac{m}{s}\right]$ & $k_{t}\left[\frac{m^{2}}{s^{s}}\right]$ & $\Lambda[m]$ \\
\hline 20\% stator height & 144.27 & 9.96 & 0.0031 \\
50\% stator height & 131.57 & 25.47 & 0.0047 \\
$\mathbf{5 7 \%}$ stator height & $\mathbf{1 2 8 . 8 7}$ & $\mathbf{2 2 . 7 6}$ & $\mathbf{0 . 0 0 4 6}$ \\
80\% stator height & 116.60 & 31.36 & 0.0057 \\
\hline
\end{tabular}

The circumferentially averaged flow and turbulence variables for the analyzed extraction plane near the stator leading edge are shown in Fig. 10. The mean velocity and turbulence statistics for the 3D-equivalent approach, determined using radial averaging techniques described in Subsection $\mathrm{A}$. are indicated by dashed, black lines. For the remaining simulations, the mean flow and turbulence characteristics were extracted at the streamline position. The values for the simulations at $20 \%, 50 \%$, and $80 \%$ of the stator heights are indicated by the blue, green, and red lines respectively. The exact values are shown in Table 1 . The TKE, TLS, and mean flow velocity of the 3D-equivalent simulation are very similar to those of the midspan simulation.

As a result, the analytical 2D upwash velocity frequency spectra deviate only minimally for the midspan and 3D-equivalent simulations as can be seen in Fig. 11. The solid lines are spectra determined at monitoring 

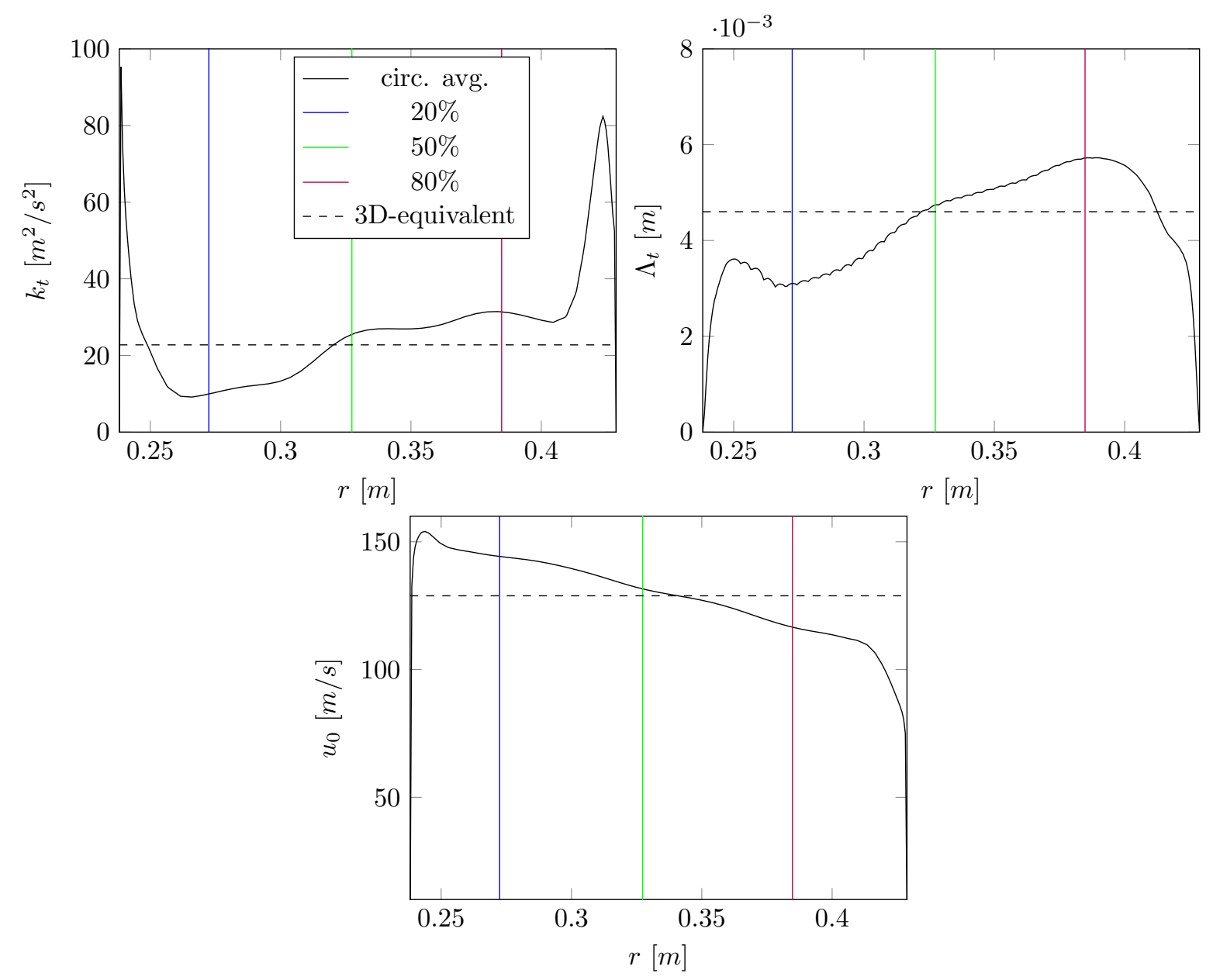

Figure 10. Circumferentially averaged turbulent kinetic energy $k_{t}$, turbulent length scale $\Lambda_{t}$, and mean flow velocity $u_{0}$ on an analysis plane near the stator leading edge
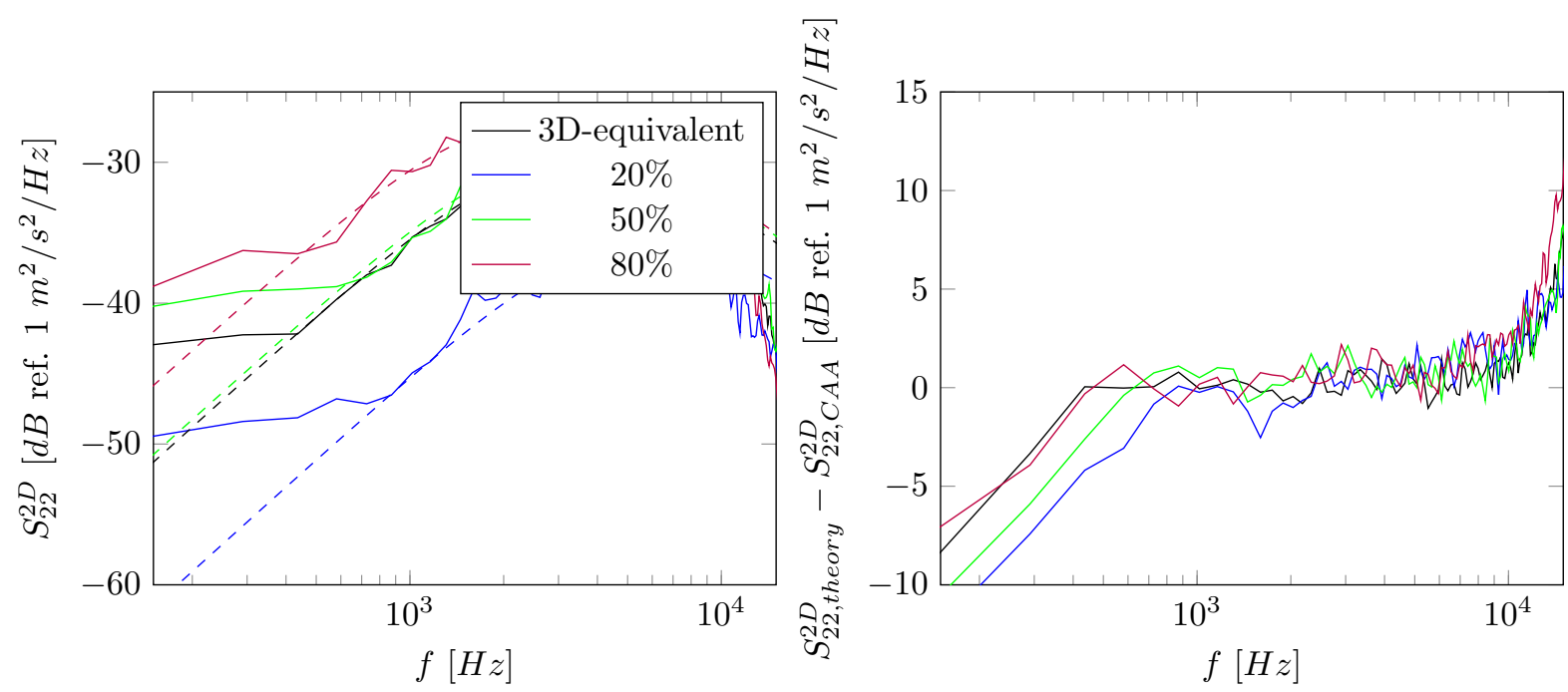

Figure 11. Comparison of velocity frequency spectra (left) and expected error margins (right) 
positions close to the stator LE in the CAA domain, while the dashed lines are analytically determined spectra. On the right hand side of Fig. 11, the difference between theoretical and realized spectra is plotted. The turbulence is realized as expected in a range between $500 \mathrm{~Hz}$ and $10 \mathrm{kHz}$ as the difference is close to zero. Below $500 \mathrm{~Hz}$, the actual levels are too high and above $10 \mathrm{kHz}$, the realized turbulence levels become too low due to the mesh resolution. The difference between prescribed and actually realized turbulence can be seen as an error margin and will be shown as such using dashed lines in subsequent diagrams showing sound power levels.

\section{E. Comparison of sound power}
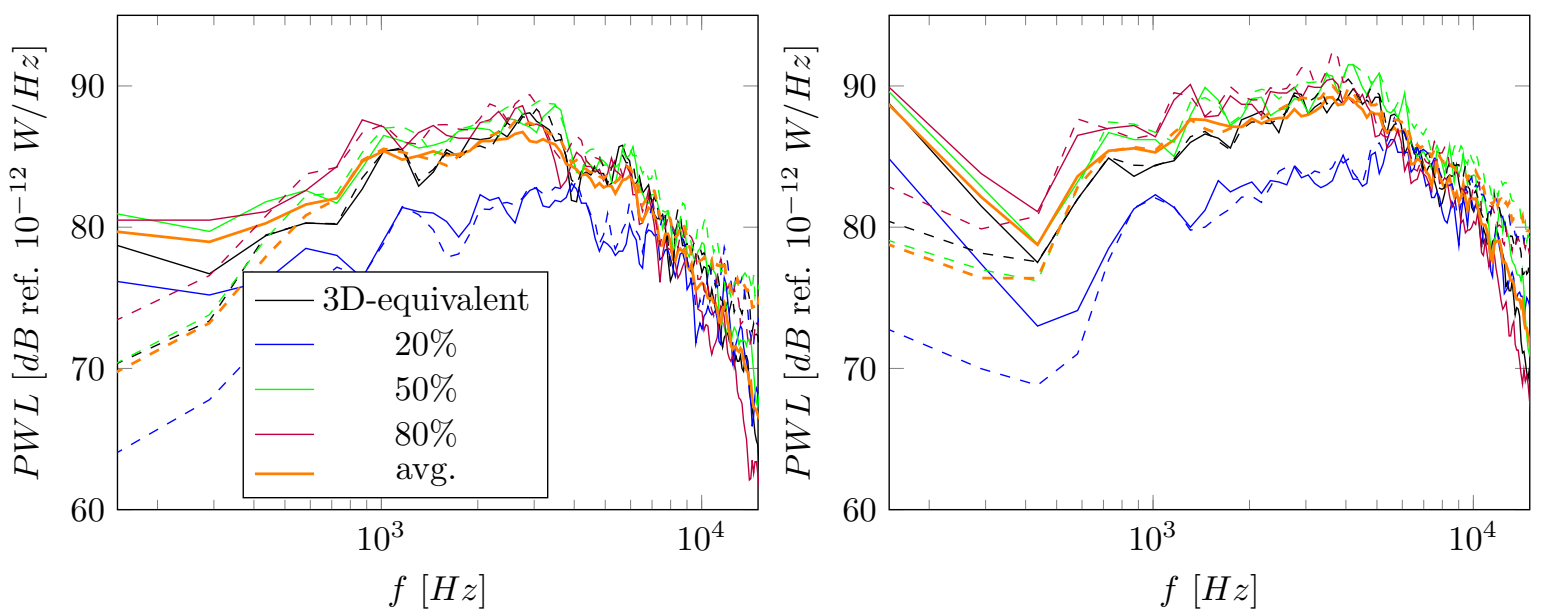

Figure 12. Comparison of upstream (left) and downstream (right) sound power level spectra: Dashed lines indicate turbulence-based error margins.

A comparison of the broadband noise predictions is discussed in terms of the sound power level spectra as shown in Fig. 12 and in terms of the overall sound power level as shown in Table 2, The sound power level spectra at 20\% stator height are significantly lower in magnitude due to the lower TKE and TLS at that position and the frequency peak is shifted to a higher frequency. The sound power level at $80 \%$ stator height is a bit higher in level at low frequencies and the frequency peak is shifted towards a lower frequency. The sound power level spectra of all three approaches (3D-equivalent - black, multi-span - orange, midspan - green) look similar in shape and level both up- and downstream of the stator vanes. This is to be expected as the turbulence and flow was similar for the 3D-equivalent and midspan approaches and the results confirm the good agreement between experimental and numerical data observed in the past 16 18 Nonetheless, larger differences are seen in the overall sound power level computed for a frequency range between $200 \mathrm{~Hz}$ and $10 \mathrm{kHz}$. The levels at $50 \%$ stator height are more than $1 \mathrm{~dB}$ higher than the levels for the 3D-equivalent approach. Averaging the intensities of the simulations at stator heights $20 \%, 50 \%$, and $80 \%$ results in overall sound power levels that are almost identical to the $3 \mathrm{D}$-equivalent results. This indicates that the multi-span approach is slightly better than the midspan approach.

Table 2. Comparison of overall sound power levels up- and downstream of the stator vanes: Numerical values are computed under consideration of error margins.

\begin{tabular}{ccc} 
& $\mathrm{OAPWL}_{\text {upstream }}[\mathrm{dB}]$ & $\mathrm{OAPWL}_{\text {downstream }}[\mathrm{dB}]$ \\
\hline 20\% stator height & 120.1 & 123.8 \\
midspan & $\mathbf{1 2 5 . 0}$ & $\mathbf{1 2 8 . 2}$ \\
$80 \%$ stator height & 124.6 & 127.6 \\
\hline multi-span & $\mathbf{1 2 3 . 6}$ & $\mathbf{1 2 6 . 9}$ \\
\hline 3D-equivalent & $\mathbf{1 2 3 . 8}$ & $\mathbf{1 2 6 . 7}$ \\
\hline
\end{tabular}




\section{Comparison to experimental data}

In this section, simulation results of the ACAT1 fan at approach conditions are compared to experimental data. The data sets are evaluated in terms of sound power and sound pressure.

A comprehensive measurement campaign for the ACAT1 fan was conducted at the UFFA test rig at AneCom Aerotest in Wildau, Germany. Acoustic data was measured at several dynamic pressure sensor arrays in the inlet, interstage, and bypass sections as well as at 25 condenser microphones positioned in a semi-arc in the far field. For a more detailed description of the experimental setup, please refer to Guérin et al! $\frac{19}{19}$ or Behn and Tapken. $\frac{33}{3}$

As the 3D-equivalent simulation delivers plausible results as shown in the previous section, all further analyses are performed using this technique. In order to ensure the best possible comparison with experimental data, a simulation of the full duct containing all 44 stator vanes was computed. The sound power level spectra of the full and quarter duct simulations are shown in Fig. 13. While the spectra remained nearly identical at higher frequencies, the spectra of the full duct simulations are smoother at lower frequencies. This can be explained by the fact that more modes are already cut-on at lower frequencies in a duct with a larger circumference. Conversely, the peaks at low frequencies of the quarter duct simulation occur when modes become cut-on. The change in overall sound power levels computed for a frequency range between $200 \mathrm{~Hz}$ and $10 \mathrm{kHz}$ is negligible.
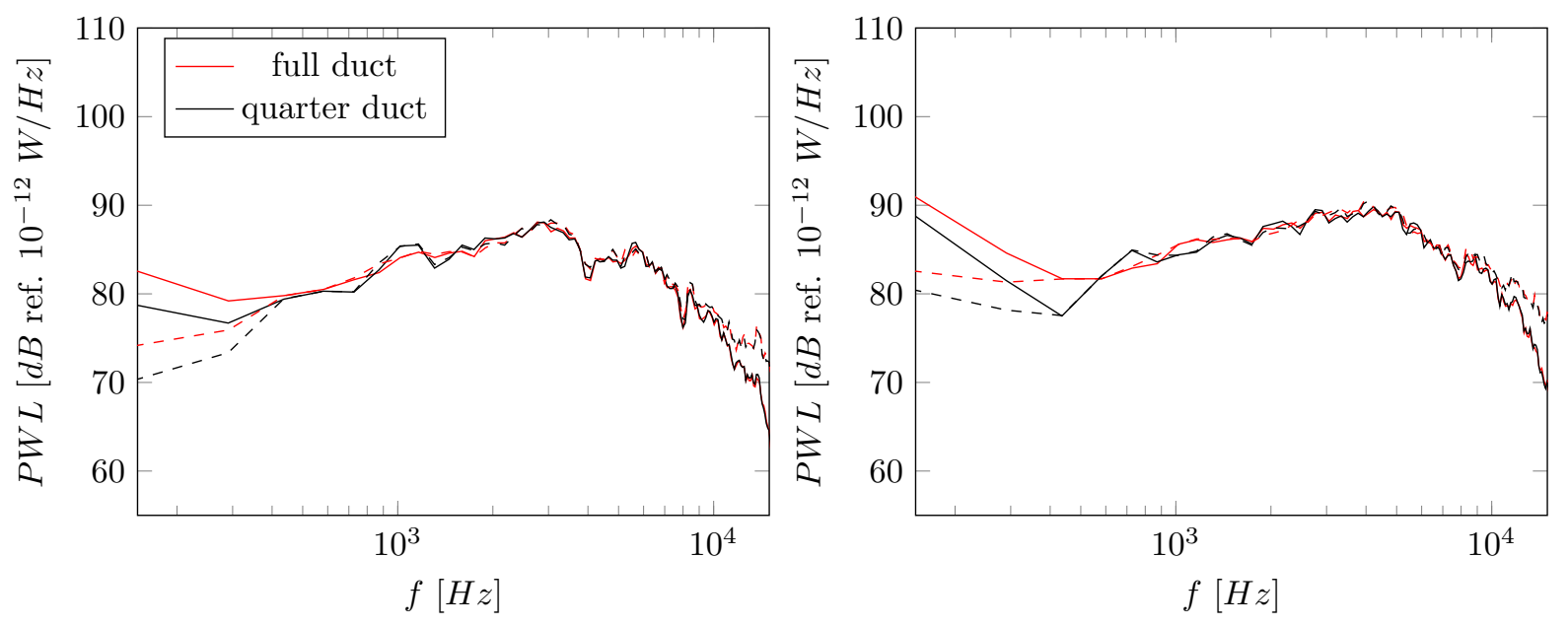

Figure 13. Comparison of sound power level spectra of quarter and full duct simulations up- (left) and downstream (right) of the stator vanes

\section{A. Sound power}

In Fig. 14, the experimental and numerical sound power level spectra are shown at positions up- and downstream of the stator vanes.

At the microphone positions in the far field, the sound power can be directly determined by integrating over a spherical surface. In the duct, different techniques exist for estimating the sound power using wallmounted microphone arrays. For this case, the determination of the sound power was based on the axial wavenumber decomposition (WND) technique, on the azimuthal mode analysis (AMA) technique, and on the Combined Axial and Azimuthal Sensor array (CAAS) method. The WND technique allows for the separation of the acoustic and hydrodynamic pressure fluctuations. ${ }^{\text {34 }}$ By resampling, the signals are synchronized with the rotor. This enables the removal of rotor-locked, i. e. tonal, components from the pressure fluctuations $\frac{35}{35}$ The sound power can then be computed by assuming an equal energy density distribution between propagating modes of the same frequency band ${ }^{[36}$ The WND data for this fan were shown and discussed in detail by Tapken et al ${ }^{37}$ The AMA technique decomposes the signal into its azimuthal mode components using a spatial Fourier transform. The sound power can be computed under the assumptions that radial modes of the same azimuthal order $m$ are incoherent and that all modes have the same propagation direction. And 

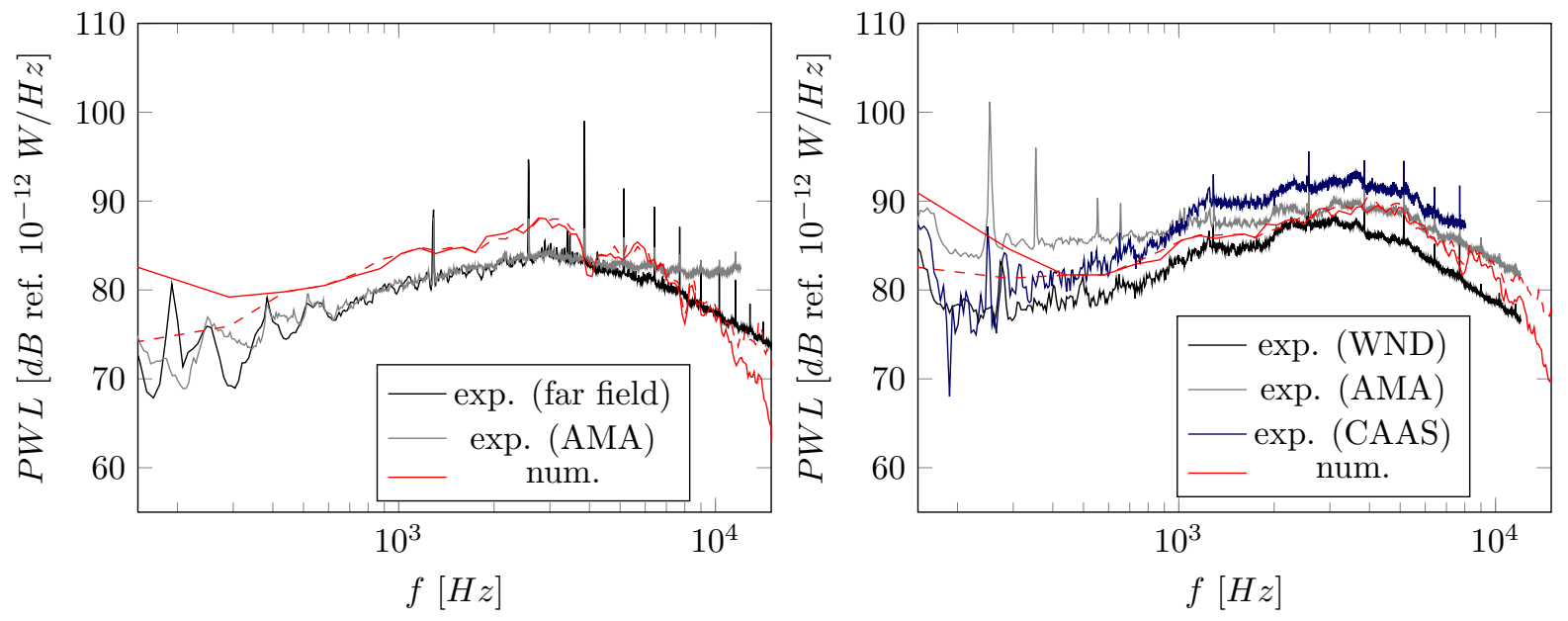

Figure 14. Comparison of experimental and numerical sound power level spectra up- (left) and downstream (right) of the stator vanes

as for the WND technique, it is assumed that the amplitudes of radial modes of the same azimuthal mode order have an equal energy distribution. Tapken et al ${ }^{37}$ performed a radial mode analysis (RMA) for the ACAT1 fan using the CAAS method. ${ }^{[38}$ This technique has the advantage that no assumption regarding the energy distribution among mode orders is necessary but no distinction between acoustic and hydrodynamic pressure fluctuations can be made. Furthermore, the CAAS-RMA method requires that all radial modes are mutually uncorrelated, which is a common assumption at least for high frequency fan broadband noise.

In the bypass, the numerical sound power levels are within the corridor of experimentally estimated data using the WND, AMA, and CAAS techniques. The AMA sound power levels are slightly higher than the WND sound power levels, particularly at lower frequencies. At very low frequencies, the CAAS sound power levels are between AMA and WND results. However, the CAAS sound power levels are even higher than the AMA sound power levels for higher frequencies. The differences in the experimental sound power levels can be mostly attributed to the techniques for estimating sound power: While the WND and AMA methods rely on an equal energy distribution assumption, the CAAS technique allows for an analysis of the radial modes and can therefore consider their realistic energy contribution. Differences between CAAS/AMA and WND sound power levels can presumably be attributed to the fact that the only the WND technique can distinguish between hydrodynamic and acoustic pressure fluctuations and a thick boundary layer resulting in some hydrodynamic pressure fluctuations can be expected at the measurement location in the bypass duct. A similar observation can be made for the measurement data at and near the inlet. At higher frequencies, the AMA sound power levels are higher than the sound power determined in the far field, which is also likely a boundary layer effect. The numerically determined sound power levels slightly overestimate the experimentally determined levels for the upstream radiated sound field. As the numerical setup cannot consider rotor shielding effects, this offset is expected. Posson et al ${ }^{39}$ used an analytical model to study rotor shielding for the NASA SDT case. They report little effect on the acoustic sound power levels at very low frequencies, about a $2 \mathrm{~dB}$ decrease at low to intermediate frequencies, and an increase of up to $1.8 \mathrm{~dB}$ at high frequencies. The latter point is a bit controversial as Hanson $\frac{40}{20}$ has reported a decrease at high frequencies due to rotor shielding.

The overall sound power levels are listed in Table 3 . The integration limits for numerical and experimental data are from $200 \mathrm{~Hz}$ up to $10 \mathrm{kHz}$. The CAAS data was thus not included as the results only range up to a maximum frequency of $8 \mathrm{kHz}$. At the inlet, the numerical simulation overestimates the overall sound power level by about $2 \mathrm{~dB}$ compared to the far field microphones due to the neglected rotor shielding effect. Downstream of the stator vanes, the numerical sound power is less than $1 \mathrm{~dB}$ smaller than estimated by the AMA technique and more than $2 \mathrm{~dB}$ higher than estimated by the WND technique. However, the overall agreement between numerical and experimental sound power levels is quite satisfactory. 
Table 3. Overall sound power levels up- and downstream of stator row at spanwise positions: Numerical values are computed under consideration of error margins.

\begin{tabular}{ccc} 
& OAPWL $_{\text {upstream }}[\mathrm{dB}]$ & OAPWL $_{\text {downstream }}[\mathrm{dB}]$ \\
\hline numerical & 123.8 & 126.8 \\
farfield mic.'s & 121.7 & \\
AMA & 122.4 & 127.4 \\
WND & & 124.3 \\
\hline
\end{tabular}

\section{B. Sound pressure}

An impression of the instantaneous sound pressure fluctuations is given in Fig. 15. The dipole characteristics of the sources at the stator leading edge is evident. Due to the stator and cascade geometries, the sound waves have different radiation patterns in the up- and downstream directions. While such a snapshot is visually pleasing, it can only give qualitative information. For a quantitative evaluation of sound pressure, experimental and numerical frequency and mode spectra are used. Note that as the fan geometry used for the numerical work has an opposing rotational direction as compared to the tested configuration, the sign of the azimuthal modes was changed for the numerical data in this section to match with the experimental data.

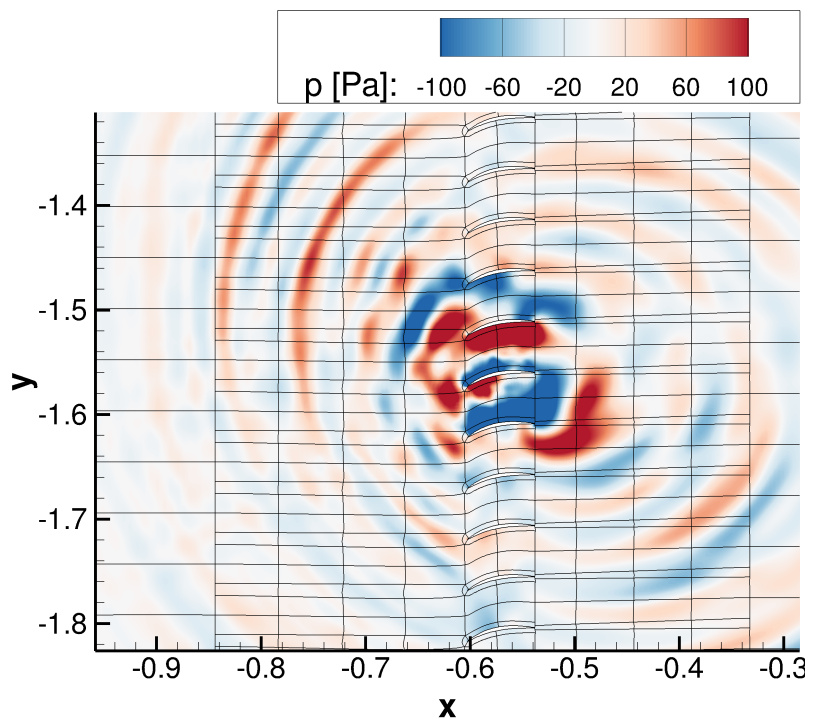

Figure 15. Instantaneous pressure fluctuations

Fig. 16 shows the comparison of the frequency spectra. The general trend is comparable to that of the sound power level spectra in Fig. 13 and the overall agreement is good.

The azimuthal mode spectra shown in Figures 17 and 18 reveal that the numerical simulation is not only able to correctly predict overall levels but also reproduces modal sound pressure patterns. A more detailed discussion of the experimental mode spectra was presented by Behn et al ${ }^{33}$ Upstream of the stator vanes, the cut-on domain are tilted with respect to the mode 0 axis as the numerical upstream microphones are positioned in the swirling flow of the interstage section and the experimental microphones are positioned in the ideally swirl-free inlet duct. Nonetheless, the clear asymmetric characteristic of the mode spectrum is captured. As the numerical simulation only considers rotor-stator-interaction noise, the dominance of the co-rotating rotor modes can be mainly attributed to that sound generation mechanism. The difference seen in the counter-rotating modes can possibly be explained by rotor shielding. Downstream of the stator vanes, both experimental and numerical data show a stronger contribution of the rotor counter-rotating modes. Both trends can be vaguely inferred from the instantaneous pressure plot in Fig. 15. Due to the leading edge 

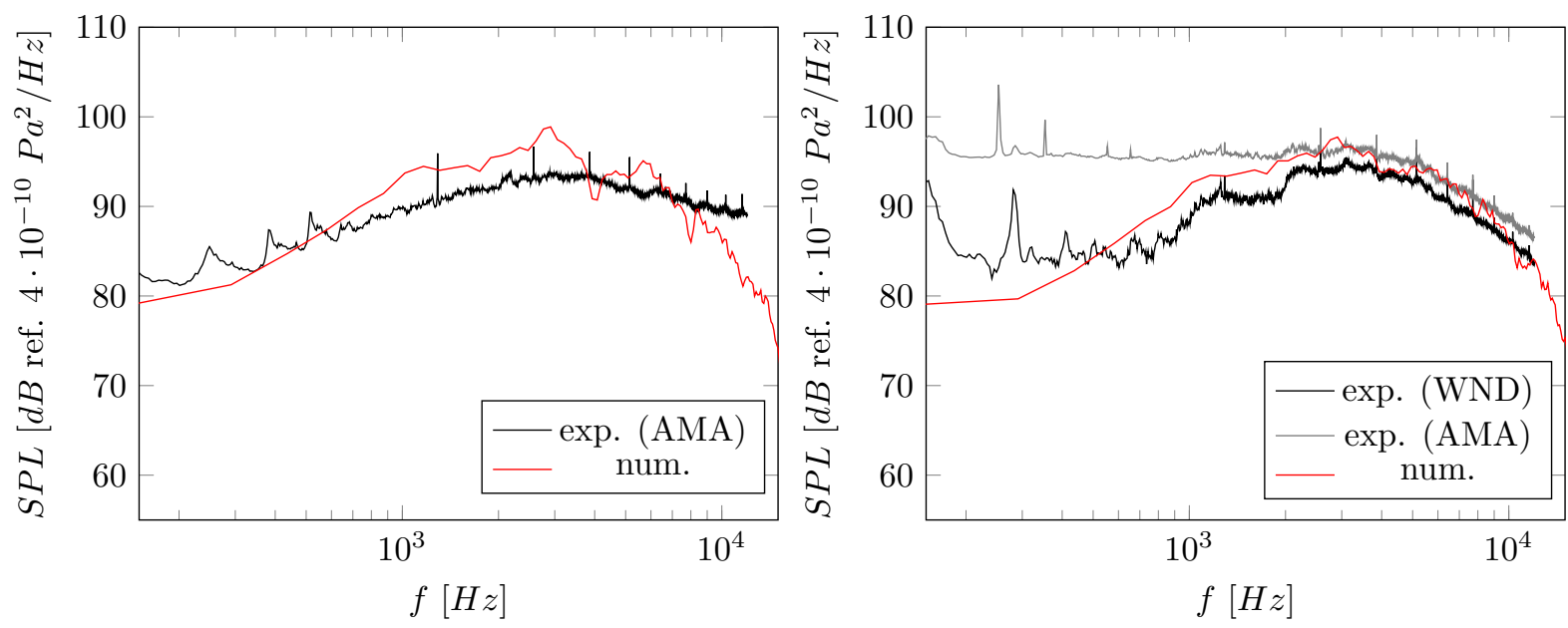

Figure 16. Comparison of experimental and numerical sound pressure level frequency spectra up- (left) and downstream (right) of the stator vanes
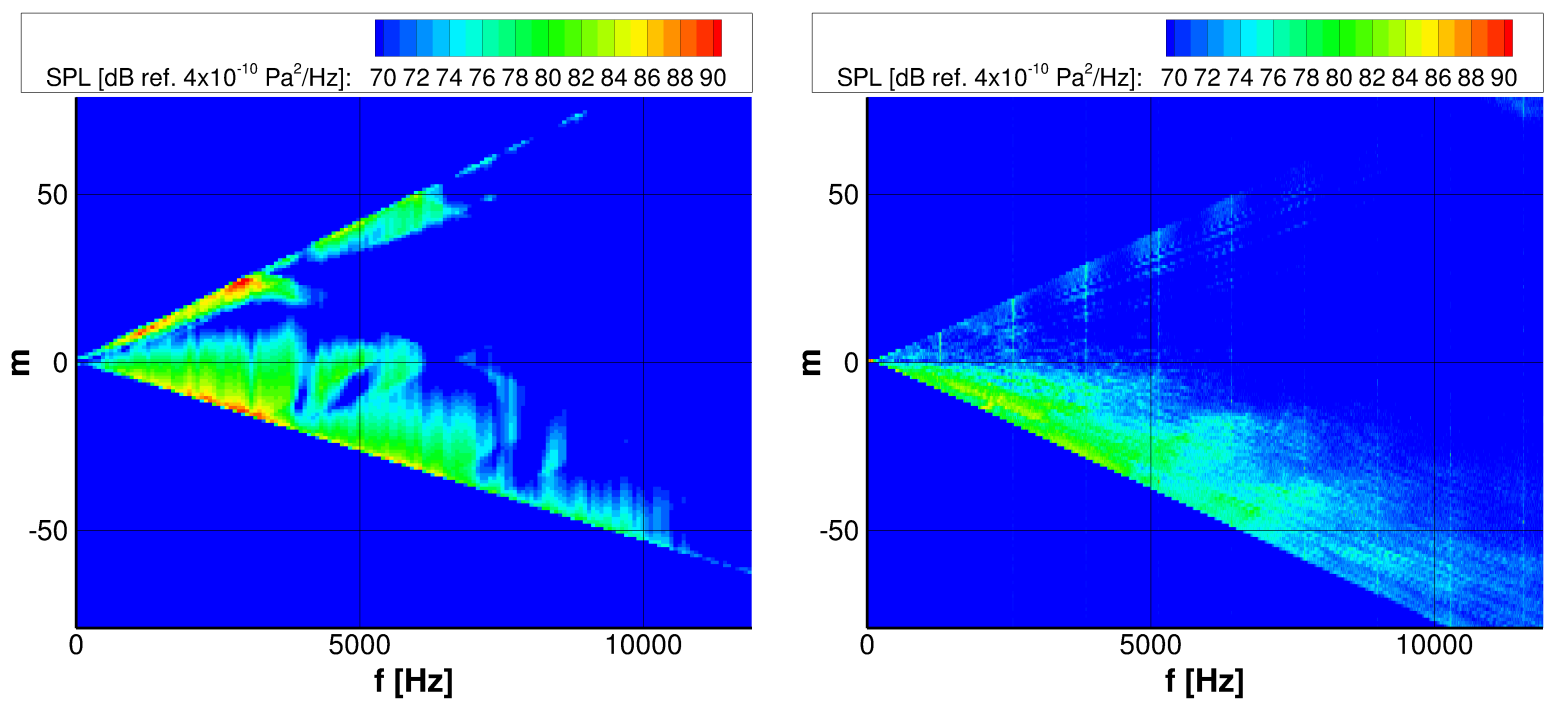

Figure 17. Comparison of the numerical (left) sound pressure level mode spectra upstream of the stator vanes and the experimental (right) sound pressure level mode spectra in the fan inlet section 

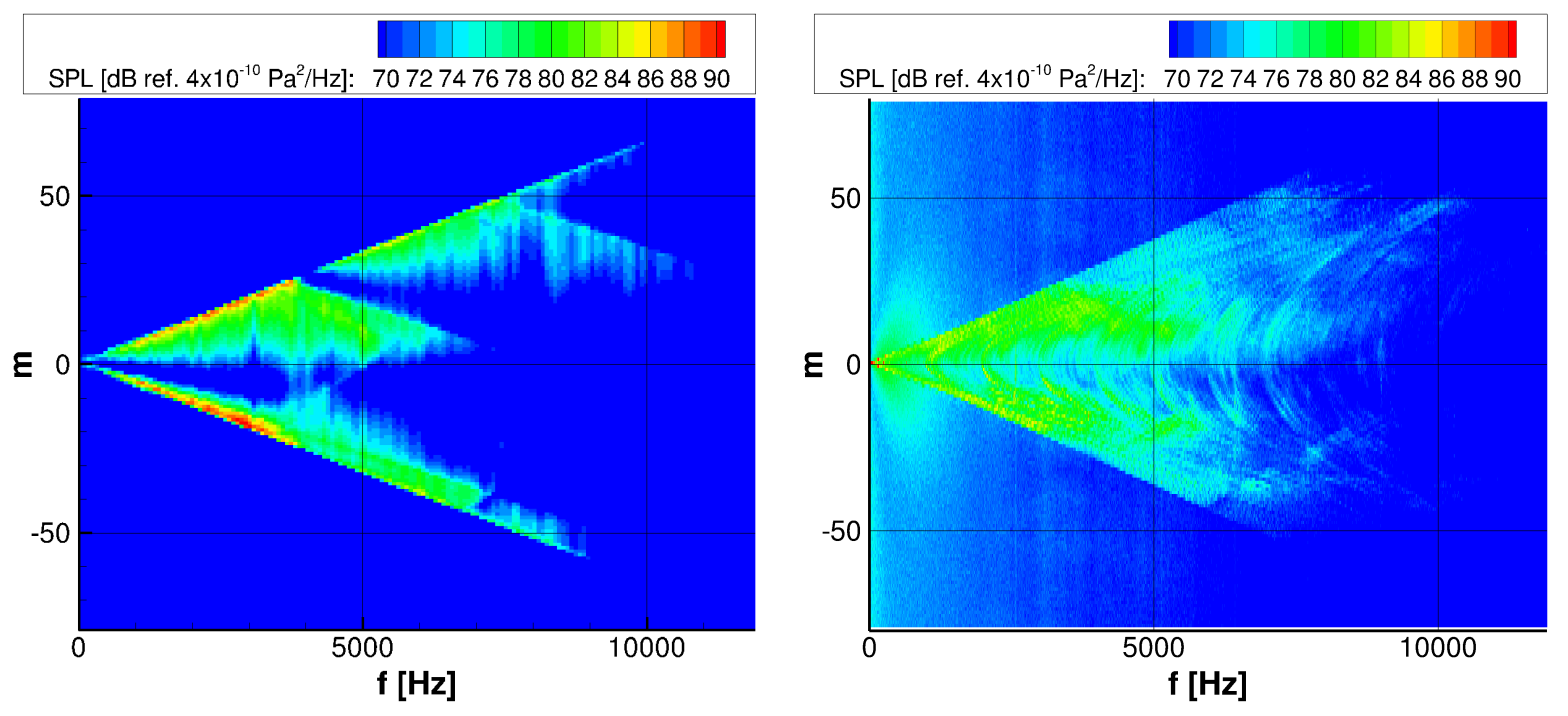

Figure 18. Comparison of numerical (left) and experimental (right) sound pressure level mode spectra downstream of the stator vanes

angle of the stator vane, pressure fluctuations originating from the suction side at the stator LE are directly propagated in the upstream direction, while pressure fluctuations from the pressure side are mostly guided by the neighboring stator vane. These reflections at the neighboring vanes cause the sound propagating in the downstream direction to be equally distributed between co- and contra-rotating modes.

\section{Conclusion}

This paper essentially consisted of two part, both of which were aimed at assessing the 2D fRPM-fan method for the prediction of fan broadband noise. In the first part, the 3D-equivalent method was introduced with the objective of setting up a 2D simulation, which is as representative as possible for the entire $3 \mathrm{D}$ duct in terms of mean flow and turbulence. This new approach was compared to the midspan and multi-span approaches, which have been successfully used in the past, to confirm its plausibility. The second part of the paper compares numerical results for the ACAT1 fan at approach to test data. Good agreement in terms of sound power and sound pressure were found. This confirms that the $2 \mathrm{D}$ simulation provides reliable results.

A new method for setting up 2D synthetic turbulence simulations was introduced. This 3D-equivalent technique has the advantage that the choice of streamline location is not arbitrary but instead relies on a detailed analysis of the flow and turbulence in the interstage domain. By using a 3D-equivalent flow and a 3D-equivalent turbulence, it is thus ensured that the simulation is as representative as possible for the entire duct for any fan geometry and any operating point. Note that this approach would have to be slightly modified if the characteristic turbulence spectrum were not shaped like a von Kármán spectrum, e. g. due to non-negligible background turbulence. In that case, the different turbulence components could be simulated by seperate simulations as proposed in the work of Kissner et al ${ }^{[7]}$ The applied multi-span approach consisted of three simulations at the arbitrarily chosen streamline positions of $20 \%, 50 \%$, and $80 \%$ of the stator height. The results in terms of the averaged sound power level spectra and overall sound power levels deviate only insignificantly from the results of the 3D-equivalent approach. However, the computational effort as well as effort required for pre- and post-processing roughly increases by a factor of three. The accuracy could be even further improved by performing simulations at even more streamline position but again at the expense of increasing cost. For the investigated case, even the simple midspan approach delivers plausible results. This is mostly due to the fact that the $50 \%$ stator height location is already fairly representative for the entire fan as shown by comparing the imposed flow and turbulence of the different simulations. However, the authors postulate that this could change when considering different fan designs and operating conditions.

The comparison to experimental data showed that reasonable agreement can be found between sound power level frequency spectra, overall sound power levels, sound pressure frequency spectra, and sound pressure mode spectra. While some deviations are expected, e. g. due to neglecting rotor shielding in the 
simulation, the overall trends and levels are reasonably well captured. The numerical results also confirm that the rotor-stator-interaction noise is indeed the dominant broadband noise contributor for the investigated fan at approach conditions for the sea level static working line.

To confirm these findings regarding fan broadband predictions using a 2D method, a 3D fRPM-fan simulation will be performed in the future. It is also envisioned to investigate anisotropic turbulence in the rotor wakes using a $3 \mathrm{D}$, cyclostationary approach.

\section{Funding Sources}

The presented work was conducted in the frame of the project TurboNoiseBB, which has received funding from the European Union's Horizon 2020 research and innovation program under grant agreement No. 690714 .

\section{Acknowledgements}

The authors thank Roland Ewert, Jürgen Dierke, and Nils Reiche (DLR) for their continued support and advice.

The authors would also like to express their gratitude to the many people of TurboNoiseBB, who have worked incredibly hard to make this test campaign a success. A special thanks goes to Azucena Pintado (AIRBUS), who provided the far-field sound power estimates shown in Fig. 14 and to Robert Meyer (DLR) and Sebastian Hakansson (DLR), who provided the turbulence spectra used for determining the inlet turbulence settings of the CFD simulation.

\section{References}

${ }^{1}$ Careta, A., Sagués, F., and Sancho, J. M., "Stochastic generation of homogeneous isotropic turbulence with well-defined spectra," Physical Review E, Vol. 48, No. 3, 1993, pp. 2279-2287.

${ }^{2}$ Ewert, R., "Broadband slat noise prediction based on CAA and stochastic sound sources from a fast random particle-mesh (RPM) method," Computers \& Fluids, Vol. 37, No. 4, 2008, pp. 369-387.

${ }^{3}$ Kraichnan, R. H., "Diffusion by a random velocity field," Physics of Fluids (1958-1988), Vol. 13, No. 1, 1970 , pp. $22-31$.

${ }^{4}$ Bechara, W., Bailly, C., Lafon, P., and Candel, S. M., "Stochastic approach to noise modeling for free turbulent flows," AIAA Journal, Vol. 32, No. 3, 1994, pp. 455-463.

${ }^{5}$ Billson, M., Eriksson, L.-E., and Davidson, L., "Jet noise prediction using stochastic turbulence modeling," 9th AIAA/CEAS Aeroacoustics Conference, Hilton Head, South Carolina, USA, 2003.

${ }^{6}$ Jarrin, N., Benhamadouche, S., Laurence, D., and Prosser, R., "A synthetic-eddy-method for generating inflow conditions for large-eddy simulations," International Journal of Heat and Fluid Flow, Vol. 27, No. 4, Aug. 2006, pp. 585-593.

${ }^{7}$ Sescu, A. and Hixon, R., "Toward low-noise synthetic turbulent inflow conditions for aeroacoustic calculations," International Journal for Numerical Methods in Fluids, Vol. 73, No. 12, 2013, pp. 1001-1010.

${ }^{8} \mathrm{Kim}$, J. W. and Haeri, S., "An advanced synthetic eddy method for the computation of aerofoil-turbulence interaction noise," Journal of Computational Physics, Vol. 287, 2015, pp. 1-17.

${ }^{9}$ Gea Aguilera, F., Gill, J., Zhang, X., Chen, X., and Nodé-Langlois, T., "Leading Edge Noise Predictions Using Anisotropic Synthetic Turbulence," 22nd AIAA/CEAS Aeroacoustics Conference, Lyon, France, 2016.

${ }^{10}$ Clair, V., Polacsek, C., Le Garrec, T., and Reboul, G., "CAA methodology to simulate turbulence-airfoil noise," 18th AIAA/CEAS Aeroacoustics Conference, Colorado Springs, Colorado, USA, 2012.

${ }^{11} \mathrm{Kim}$, J. W., Haeri, S., and Joseph, P. F., "On the mechanisms of noise reduction in aerofoil-turbulence interaction by using wavy leading edges," 21st AIAA/CEAS Aeroacoustics Conference, Dallas, Texas, USA, 2015.

${ }^{12}$ Gea-Aguilera, F., Gill, J., Angland, D., and Zhang, X., "Wavy Leading Edge Airfoils Interacting with Anisotropic Turbulence," 23rd AIAA/CEAS Aeroacoustics Conference, Denver, Colorado, USA, 2017.

${ }^{13}$ Reboul, G., Cader, A., Polacsek, C., Le Garrec, T., Barrier, R., and Nasr, N. B., "CAA Prediction of Rotor-Stator Interaction Using Synthetic Turbulence: Application to a Low-Noise Serrated OGV," 23rd AIAA/CEAS Aeroacoustics Conference, Denver, Colorado, USA, 2017.

${ }^{14}$ Gill, J., Zhang, X., Joseph, P., and Nodé-Langlois, T., "Reduced dimension modeling of leading edge turbulent interaction noise," 20th AIAA/CEAS Aeroacoustics Conference, Atlanta, Georgia, USA, 2014.

${ }^{15}$ Cader, A., Polacsek, C., Garrec, T. L., Barrier, R., Benjamin, F., and Jacob, M. C., "Numerical prediction of rotor-stator interaction noise using 3D CAA with synthetic turbulence injection," 24th AIAA/CEAS Aeroacoustics Conference, Atlanta, Georgia, USA, 2018.

${ }^{16}$ Wohlbrandt, A., Kissner, C., and Guérin, S., "Impact of cyclostationarity on fan broadband noise prediction," Journal of Sound and Vibration, Vol. 420, April 2018, pp. 142-164.

${ }^{17}$ Kissner, C. A., Wohlbrandt, A. M., and Guérin, S., "Enhanced Fan Broadband Noise Prediction Based on a 2D Synthetic Turbulence Method," 24th AIAA/CEAS Aeroacoustics Conference, Atlanta, Georgia, USA, 2018. 
${ }^{18}$ Kissner, C. A. and Guérin, S., "Influence of Wake and Background Turbulence on Predicted Fan Broadband Noise," submitted to the AIAA Journal, 2019.

${ }^{19}$ Guérin, S., Kissner, C., Kajasa, B., Jaron, R., Behn, M., Hakansson, S., Pardowitz, B., Tapken, U., Meyer, R., and Enghardt, L., "Noise prediction of the ACAT1 fan with a RANS-informed analytical method: success and challenge," 25th AIAA/CEAS Aeroacoustics Conference, Delft, Netherlands, 2019.

${ }^{20}$ Siefert, M. and Ewert, R., "Sweeping Sound Generation in Jets Realized with a Random Particle-Mesh Method," 15th AIAA/CEAS Aeroacoustics Conference, Miami, Florida, USA, 2009.

${ }^{21}$ Wohlbrandt, A., Hu, N., Guérin, S., and Ewert, R., "Generalised turbulence spectra for broadband noise predictions with the Random Particle Mesh method," Computers and Fluids, Vol. 132, 2016, pp. 46-50.

${ }^{22}$ Delfs, J. W., Bauer, M., Ewert, R., Grogger, H., Lummer, M., and Lauke, T., "Numerical Simulation of Aerodynamic Noise with DLR's aeroacoustic code PIANO," Tech. rep., German Aerospace Center (DLR), Braunschweig, Jan. 2008.

${ }^{23} \mathrm{Hu}$, F. Q., Hussaini, M. Y., and Manthey, J. L., "Low-dissipation and low-dispersion Runge - Kutta schemes for computational acoustics," Journal of Computational Physics, Vol. 124, No. 1, 1996, pp. 177-191.

${ }^{24}$ Tam, C. K. and Webb, J. C., "Dispersion-relation-preserving finite difference schemes for computational acoustics," Journal of Computational Physics, Vol. 107, No. 2, 1993, pp. 262-281.

${ }^{25}$ Ewert, R., Dierke, J., Neifeld, A., and Moghadam, S. A., "Linear-and Non-Linear Perturbation Equations with Relaxation Source Terms for Forced Eddy Simulation of Aeroacoustic Sound Generation," 20th AIAA/CEAS Aeroacoustics Conference, Atlanta, Georgia, USA, 2014.

${ }^{26}$ Becker, K., Heitkamp, K., and Kügeler, E., "Recent Progress in a Hybrid Grid CFD Solver for Turbomachinery Flows," Proc.V European Conference on Computational Fluid Dynamics ECCOMAS CFD 2010, Lisbon, Portugal, 2010.

${ }^{27}$ Cécora, R.-D., Radespiel, R., Eisfeld, B., and Probst, A., "Differential Reynolds-stress modeling for aeronautics," AIAA Journal, Vol. 53, No. 3, 2015, pp. 739-755.

${ }^{28}$ Menter, F. R., "Two-equation eddy-viscosity turbulence models for engineering applications," AIAA Journal, Vol. 32, No. 8, 1994, pp. 1598-1605.

${ }^{29}$ Tyler, J. M. and Sofrin, T. G., "Axial flow compressor noise studies," Tech. rep., SAE Technical Paper, 1962.

${ }^{30}$ Wohlbrandt, A., Weckmüller, C., and Guérin, S., "A robust extension to the triple plane pressure mode matching method by filtering convective perturbations," International Journal of Aeroacoustics, Vol. 15, No. 1-2, 2016, pp. 41-58.

${ }^{31}$ De Roeck, W., Desmet, W., Baelmans, M., and Sas, P., "An overview of high-order finite difference schemes for computational aeroacoustics," Proceedings of the International Conference on Noise and Vibration Engineering, Citeseer, 2004, pp. $353-368$.

${ }^{32}$ Amiet, R. K., "Acoustic radiation from an airfoil in a turbulent stream," Journal of Sound and Vibration, Vol. 41, No. 4, Aug. 1975, pp. 407-420.

${ }^{33}$ Behn, M. and Tapken, U., "Investigation of Sound Generation and Transmission Effects Through the ACAT1 Fan Stage using Compressed Sensing-based Mode Analysis," 25th AIAA/CEAS Aeroacoustics Conference, Delft, Netherlands, 2019.

${ }^{34}$ Tapken, U., Pardowitz, B., and Behn, M., "Radial mode analyis of fan broadband noise," 23rd AIAA/CEAS Aeroacoustics Conference, Denver, Colorado, USA, 2017.

${ }^{35}$ Behn, M., Pardowitz, B., and Tapken, U., "Separation of tonal and broadband noise components by cyclostationary analysis of the modal sound field in a low-speed fan test rig," Fan2018, Darmstadt, Germany, 2018.

${ }^{36}$ Joseph, P., Morfey, C. L., and Lowis, C. R., "Multi-mode sound transmission in ducts with flow," Journal of Sound and Vibration, Vol. 264, No. 3, 2003, pp. 523-544.

${ }^{37}$ Tapken, U., Behn, M., Spitalny, M., and Pardowitz, B., "Radial mode breakdown of the ACAT1 fan broadband noise generation in the bypass duct using a sparse sensor array," 25th AIAA/CEAS Aeroacoustics Conference, Delft, Netherlands, 2019.

${ }^{38}$ Tapken, U., Gutsche, D., and Enghardt, L., "Radial mode analysis of broadband noise in flow ducts using a combined axial and azimuthal sensor array," 20th AIAA/CEAS Aeroacoustics Conference, Atlanta, Georgia, USA, 2014.

${ }^{39}$ Posson, H. and Moreau, S., "Effect of Rotor Shielding on Fan-Outlet Guide Vanes Broadband Noise Prediction," AIAA Journal, Vol. 51, No. 7, 2013, pp. 1576-1592.

${ }^{40}$ Hanson, D. B., "Broadband Noise of Fans - With Unsteady Coupling Theory to Account for Rotor and Stator Reflection/Transmission Effects," Tech. rep., Nov. 2001. 